\title{
Free gravity waves and balanced dynamics
}

Article

Published Version

Wirosoetisno, D., Shepherd, T. G. and Temam, R. M. (2002) Free gravity waves and balanced dynamics. Journal of the Atmospheric Sciences, 59. pp. 3382-3398. ISSN 1520-0469 doi: https://doi.org/10.1175/15200469(2002)059<3382:FGWABD>2.0.CO;2 Available at https://centaur.reading.ac.uk/32215/

It is advisable to refer to the publisher's version if you intend to cite from the work. See Guidance on citing.

To link to this article DOI: http://dx.doi.org/10.1175/1520-

0469(2002)059<3382:FGWABD>2.0.CO;2

Publisher: American Meteorological Society

All outputs in CentAUR are protected by Intellectual Property Rights law, including copyright law. Copyright and IPR is retained by the creators or other copyright holders. Terms and conditions for use of this material are defined in the End User Agreement.

www.reading.ac.uk/centaur 
Central Archive at the University of Reading

Reading's research outputs online 


\title{
Free Gravity Waves and Balanced Dynamics
}

\author{
D. WirOSOETISNO \\ Department of Physics, University of Toronto, Toronto, Ontario, Canada, and Institute for Scientific Computing and \\ Applied Mathematics, Indiana University at Bloomington, Bloomington, Indiana, and Department of Mathematics and Statistics, \\ University of Edinburgh, Edinburgh, United Kingdom \\ T. G. SHEPHERD \\ Department of Physics, University of Toronto, Toronto, Ontario, Canada \\ R. M. TEMAM \\ Institute for Scientific Computing and Applied Mathematics, Indiana University at Bloomington, Bloomington, Indiana, and \\ Laboratoire d'Analyse Numérique, Université Paris-Sud, Orsay, France
}

(Manuscript received 31 July 2001, in final form 14 May 2002)

ABSTRACT

\begin{abstract}
It is shown how a renormalization technique, which is a variant of classical Krylov-Bogolyubov-Mitropol'skii averaging, can be used to obtain slow evolution equations for the vortical and inertia-gravity wave components of the dynamics in a rotating flow. The evolution equations for each component are obtained to second order in the Rossby number, and the nature of the coupling between the two is analyzed carefully. It is also shown how classical balance models such as quasigeostrophic dynamics and its second-order extension appear naturally as a special case of this renormalized system, thereby providing a rigorous basis for the slaving approach where only the fast variables are expanded. It is well known that these balance models correspond to a hypothetical slow manifold of the parent system; the method herein allows the determination of the dynamics in the neighborhood of such solutions. As a concrete illustration, a simple weak-wave model is used, although the method readily applies to more complex rotating fluid models such as the shallow-water, Boussinesq, primitive, and 3D Euler equations.
\end{abstract}

\section{Introduction}

It is well known that in the atmosphere as well as in the oceans the two most important components of the dynamics are vortical motion and inertia-gravity waves. At the synoptic scale and larger, the vortical motions predominate, giving rise to a nearly "balanced" dynamics. If the dynamics were perfectly balanced (in the sense of section 3 below), the description and evolution of the system would be determinable from a reduced set of variables, usually taken to be the potential vorticity. The real atmosphere and oceans, however, do contain a certain amount of seemingly freely propagating gravity waves, which can be regarded as a component "orthogonal" to the balanced dynamics. Comparatively little is known about the interactions between

Corresponding author address: Prof. T. G. Shepherd, Dept. of Physics, University of Toronto, 60 St. George St., Toronto, ON M5S 1A7, Canada.

E-mail: tgs@atmosp.physics.utoronto.ca these two components, since the usual construction of balance models postulates that these free gravity waves do not exist.

A number of different methods have been used to derive balance models. In the most common and arguably most systematic method, the governing equations are scaled and a small parameter (usually related to timescale separation) is introduced. The fast variables are then expanded in powers of the small parameter, and a hierarchy of balance models is obtained; this corresponds to the "slow time expansion" or "slaving" procedure (Warn et al. 1995), which is discussed in detail below. When one takes the Rossby number as the small parameter on the $f$ plane, possibly with weak topography, the leading nontrivial model in the hierarchy is the familiar quasigeostrophic model (Pedlosky 1987).

Many other approaches have been used to obtain slow solutions to systems that support fast oscillations. In the early days, balance models were derived more or less intuitively (Charney 1948, 1955); various initialization 


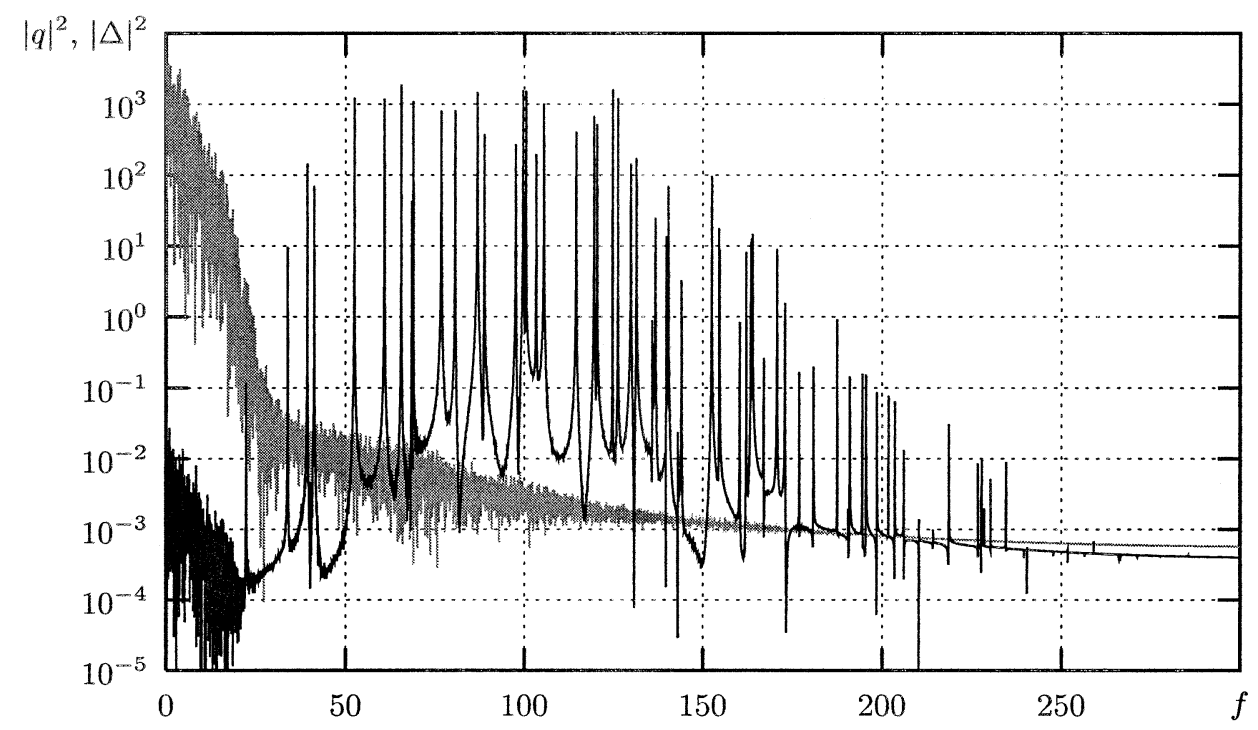

FIG. 1. Power spectra at $x=(0,0)$ of potential vorticity $q$ (gray line) and divergence $\Delta$ (black line) of the weak-wave model (see sections 4 and 5 for details) taken over $0 \leq t \leq 204.7$, with frequency $f$ expressed in terms of $t^{-1}$. Here, $B=0.5$ and Ro $=0.1$, so the minimum gravity wave frequency is at $f$ $=\varepsilon^{-1} \sim 22.4$. Separation of the fast and slow motion is evident: $q$ has power predominantly in the low frequencies, with a sharp decrease near $f \sim \varepsilon^{-1}$; meanwhile, the power of $\Delta$ resides mainly in the strong peaks at the frequencies of the gravity waves, although there is also what is presumably a slaved component at low frequencies, mirroring $q$ but much weaker.

procedures, including the bounded derivative method, were used operationally (Baer and Tribbia 1977; Machenhauer 1977; Kreiss 1979); iterative methods have also been used to derive higher-order balance models (e.g., Allen 1993; McIntyre and Norton 2000). Some of these methods are closely related, if not demonstrably equivalent, to the slaving method of Warn et al. (1995); in fact, we are planning a future article devoted to this issue.

These asymptotic procedures work because of the timescale separation between the fast and slow dynamics. Inspection of Fig. 1 (obtained from an uninitialized numerical simulation using the model described in section 4 below) reveals that the power of the slow variable $q$ is confined mostly to frequencies below $f=\varepsilon^{-1}$, where $\varepsilon$ is the ratio of fast to slow timescale, while most of the power of the fast variable $\Delta$ resides in sharp peaks having frequencies greater than or equal to $f=\varepsilon^{-1}$.

It should be noted that for reasonably long times, classical balance can be very effective (Wirosoetisno 1999). In the top panel of Fig. 2, we show how successively higher-order initializations of the ageostrophic variables can eliminate most of their fast oscillations. Thus, when properly initialized (i.e., with properly chosen initial conditions), the "fast" ageostrophic variables evolve slowly for some time. Much of this slow evolution is slaved to the slow variables, as further underlined in the bottom panel: for times of up to several tens of eddy turnaround times, only a very small fraction (of order $10^{-6}$ in terms of power) of the ageostrophic var- iable is unaccounted for by $O\left(\varepsilon^{3}\right)$ classical balance; higher-order balance may reduce this even further.

If such a procedure were to converge, one would find an invariant "slow manifold" to which any solution starting on it is confined for all time. It is now accepted wisdom, however, that such an invariant manifold is unlikely to exist (Vautard and Legras 1986; Lorenz and Krishnamurthy 1987; Lorenz 1992; Wirosoetisno 1999). Warn (1997) has argued that the asymptotic nature of the procedure (or of the concept of balance in general) is related to the impossibility to (find a set of variables that would) eliminate the spectral overlap that is evident in Fig. 1.

Given the impossibility of finding exact balance, a natural question to ask is: When the unbalanced motion is nonzero, what is the nature of its interaction with the balanced flow? Several studies have been done to address this problem. Ford (1994a,b,c) has obtained analytical estimates, and numerical confirmation, on the amplitude of gravity waves radiated away by certain vortical flows. Polvani et al. (1994) have found numerically that these estimates appear to apply in more general cases, where the mathematical theory is, strictly speaking, no longer valid. A number of purely numerical investigations have also been carried out, mostly with the conclusion that gravity waves are inevitably generated from balanced initial conditions (Farge and Sadourny 1989; Yuan and Hamilton 1994; Yavneh and McWilliams 1994). However, the numerical studies are difficult to interpret because the initial balance is only 

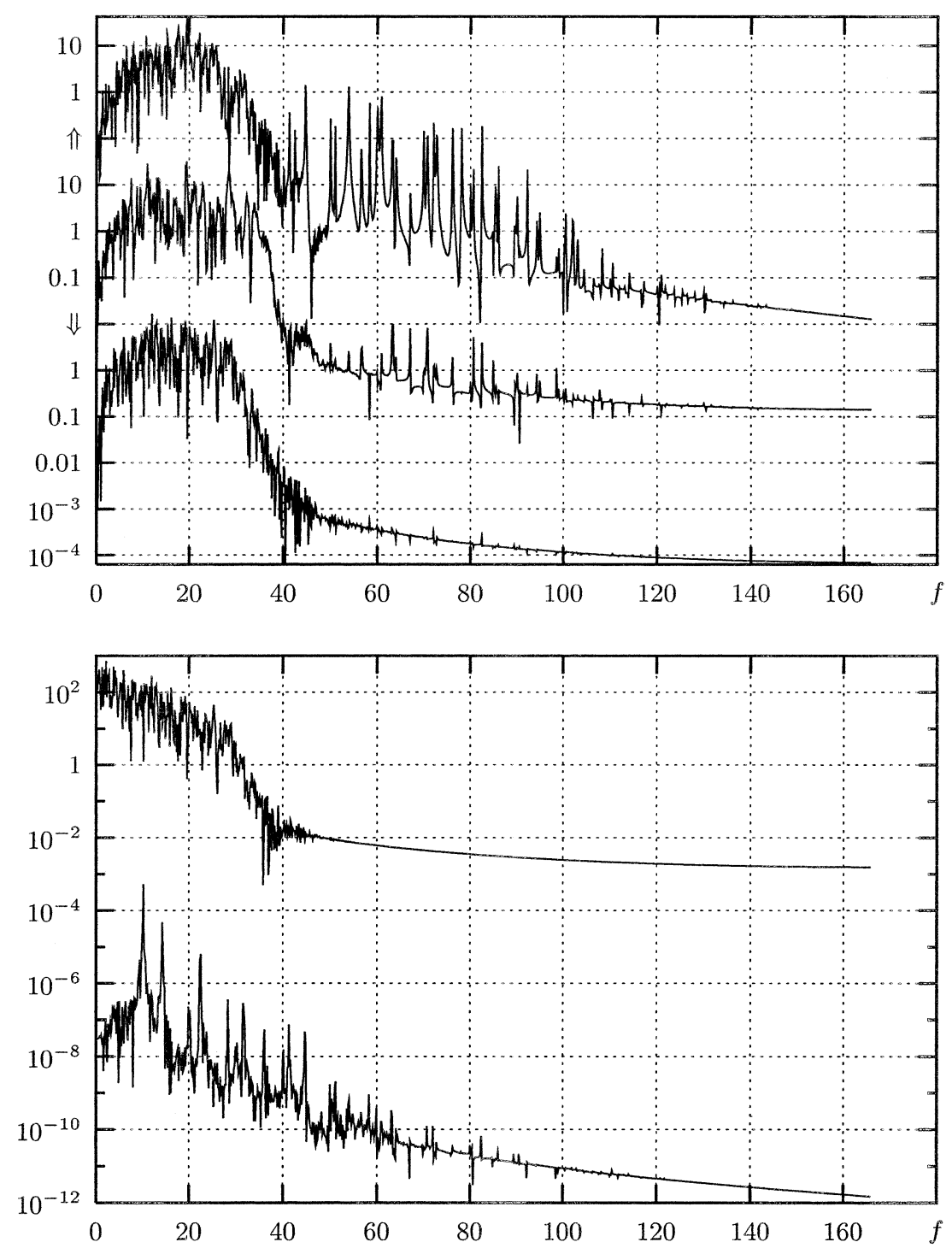

FIG. 2. Power spectra at $x=(0,0)$, with Ro $=0.5, B=0.2$, and $\varepsilon^{-1} \approx 10.2$. The numerical model is as described in section 5 except the resolution is $29^{2}$, the aspect ratio $a=1$, and in (5.1) $k_{0}=3$. The frequency spectrum is taken over $0 \leq t \leq 38.8$. (top) Spectra of $\Delta$, without initialization, that is, only with geostrophic balance (top curve, shifted up by $10^{3}$ ), with $O(\varepsilon)$ slaving initialization (middle curve, in place), and with $O\left(\varepsilon^{3}\right)$ initialization (bottom curve, shifted down by $10^{-3}$ ). (bottom) Spectra of $q$ (upper curve) and $\Delta-U_{3}^{\Delta}$ (lower curve), that is, the third-order estimate of the unslaved, or free, component of divergence; both in place, with third-order slaving initialization. The third-order estimate of the unslaved divergence reveals the free inertia-gravity waves that are submerged beneath the slaved (balanced) spectrum in the bottom curve of the top panel. Hence, there is a separation of the fast and slow dynamics even when their spectra strongly overlap.

approximate, and there is the very real possibility that a more accurate initial balance would reduce the gravity wave generation.

To better understand this problem, in this paper we employ an asymptotic theory based on the renormalization method to obtain slow evolution equations for both the amplitude of the "free" gravity waves and the balanced vortical motion. The coupling between these two components can be explicitly identified, thus show- ing how unbalanced motion can be generated from vortical flows and how the dynamics of the latter is affected by nonzero gravity waves.

As described in detail below, classical balance turns out to be a special case of our renormalized system where, interestingly, the renormalization procedure has no effect. Using a combination of the renormalized equations and error bounds on their solutions, it is possible to predict the stability of these classical balance 
solutions. (More generally, the renormalization approach makes it possible to estimate the stability of the asymptotic solutions of a class of singular perturbation problems.)

The idea of renormalization, which dates back to the 1940s, is hardly new, with its application to singular perturbation theory being slightly more recent [cf. the monographs of Nayfeh (1973), and of Goldenfeld (1992), and the paper by Chen et al. (1996)]. The form used in this paper is a development of the work of Moise et al. (1998), Ziane (2000), Moise and Temam (2000), and Moise and Ziane (2001). The applications treated in the last two papers are of interest to geophysical fluid dynamics (GFD): slightly compressible flows and the Galerkin truncation of the Navier-Stokes equations.

What amounts to our first-order renormalized equations have been investigated previously by a number of authors in various GFD contexts (cf. e.g., Embid and Majda 1996; Chemin 1997; Gallagher 1998; Embid and Majda 1998; Majda and Embid 1998; Babin et al. 2000, 2001) based on the work of Schochet (1994), which is closely related to the method employed here. Mention should also be made of the work of Callet (1997), who analyzed four-wave resonances-these correspond to isolated interactions in our second-order renormalized system. Apart from presenting a possibly more transparent derivation of these results, the systematic expansion presented in this work can be readily [at least formally for partial differential equations (PDEs)] extended to higher orders while at the same time keeping a clear relationship to higher-order classical balance models.

To emphasize the geophysical relevance, we shall refer to the well-known rotating shallow-water model as an illustration to the abstract derivation. However, in the worked example of section 4, a simpler model, the weak-wave model of Nore and Shepherd (1997), will be used since it significantly reduces the amount of computation.

The rest of this paper is structured as follows. In section 2 we describe the renormalization method and set the notation used in the rest of this paper. The close connection between the renormalization solution and that obtained by the slaving method is presented in section 3. Section 4 contains a worked example of the formalism of the previous two sections, and numerical simulations of this model are presented in section 5. A discussion concludes the paper.

\section{The renormalization method}

In this section, we consider a dynamical system of the form

$$
\frac{d u}{d s}+L u=\varepsilon \mathcal{F}(u), \quad u(0)=u_{0},
$$

where $\mathcal{F}(u)$ is a polynomial function of its argument with $\mathcal{F}(0)=0, L$ is a linear operator, and $\varepsilon$ is a small parameter. Systems of this form arise from the spectral discretization of virtually all GFD models in their unforced form (with minor modification, the formulation below can handle time-quasi-periodic forcing). In general $\mathcal{F}$ may depend on $\varepsilon$, but this case adds no essential difficulty apart from the amount of computation needed, so we will not discuss this case further in what follows. For the formal development in most of this section we do not assume that the system (2.1) is finite-dimensional. The system could be, say, a system of partial differential equations in Fourier representation. Some additional assumptions would however be needed in the PDE case; see the remarks at the end of this section.

For concreteness, we use the rotating shallow-water equations on the $f$ plane (SWE for short) to provide the geophysical illustration of the formalism, which, however, is equally applicable to many other systems as well. In this context, the dependent variable $u$ can be regarded as representing the velocity (or its derivatives) and surface height, $L$ is the operator corresponding to the inertia-gravity waves, and (taking the rotational Froude number to be 1) $\varepsilon$ is the Rossby number. In this section we work on the fast time $s=t / \varepsilon$ and seek to rewrite the equations, by a change of variable, in a form where the fast part $L u$ disappears. The connection between this expansion and the slow time $t$ expansion, which yields the classical balance models, is described in section 3 below.

The requirement to work with a discrete (but not necessarily finite) set of equations appears to be essential for the method; it will be apparent from the development below why, while classical balance models can be derived strictly in the continuous formulation, one needs a discrete spectral formulation as soon as free gravity waves are considered.

Before we begin, all series solutions (and the slow manifold $\mathcal{S}_{\text {rg }}$ and $\mathcal{S}_{\text {slav }}$ ) in this paper are to be understood in the asymptotic sense; we make no claims as to the convergence of any expansion as $n \rightarrow \infty$.

Defining a new variable $v:=e^{L s} u$, we can write our system in the equivalent form

$$
\frac{d v}{d s}=\varepsilon F(v, s), \quad v(0)=u_{0}
$$

where $F(v, s)=e^{L s} \mathcal{F}\left(e^{-L s} v\right)$. In the rest of this paper, we will make use of both forms, (2.1) and (2.2), whichever is more convenient.

The first step is standard: we expand the variable $v$ in powers of $\varepsilon$,

$$
v(s)=v^{0}(s)+\varepsilon v^{1}(s)+\varepsilon^{2} v^{2}(s)+\cdots
$$

and solve the resulting system of equations order by order. Under this expansion, $F(v, s)$ on the right-hand side of (2.2) becomes 


$$
\begin{aligned}
F(v, s)= & F\left(v^{0}, s\right)+\varepsilon F^{\prime}\left(v^{0}, s\right) \cdot v^{1}(s) \\
& +\varepsilon^{2}\left[F^{\prime}\left(v^{0}, s\right) \cdot v^{2}(s)\right. \\
& \left.\quad+\frac{1}{2} F^{\prime \prime}\left(v^{0}, s\right) \cdot v^{1}(s) \otimes v^{1}(s)\right]+O\left(\varepsilon^{3}\right)
\end{aligned}
$$

where the primes on $F$ denote differentiation with respect to its first argument. ${ }^{1}$ Here and elsewhere, we may suppress writing the $s$ dependence in functions when no confusion can arise.

The leading-order solution is easily obtained. Since

$$
\frac{d v^{0}}{d s}=0
$$

we have $v^{0}(s)=$ constant $=: v_{0}$ [note that it may be advantageous to choose $v_{0} \neq v(0)=u_{0}$; see remark following (2.10) below]. Equivalently, we have $u^{0}(s)=e^{-L s} v_{0}$. In our SWE illustration, this simply says that the gravity waves are linear oscillators at leading order while the vortical modes-for which $e^{L s}$ is the identity-are frozen on the fast timescale. To compute the next-order terms, we need some more notation.

Following Bogolyubov and Mitropol'skii (1958), for an arbitrary function $G(w, s)$ that can be written in the form

$$
G(w, s)=\sum_{j} e^{i \nu_{j} s} f_{j}(w)
$$

we define a pair of operators. [Note that $F(v, s)$ above can be written in this form, even with the addition of time-quasi-periodic forcing, $\mathcal{F}$ being a polynomial.] The averaging operator, denoted by an overbar, picks out the constant part of $G(w, s)$, that is, the part that does not depend explicitly on time:

$$
\overline{G(w)}:=\sum_{\nu_{j}=0} f_{j}(w) .
$$

The integrating operator, denoted by an underbar, is

$$
\underline{G(w, s)}:=\sum_{\nu_{j} \neq 0} \frac{1}{i \nu_{j}} e^{i \nu_{j} s} f_{j}(w) .
$$

With these definitions, we have

$$
G(w, s)=\overline{G(w)}+\frac{\partial}{\partial s} \underline{G(w, s)},
$$

as well as $\overline{G(w, s)}=0$ (note that the two operators commute). We stress that the averaging and integrating operators act on the explicit time dependence of the function $G(w, s)$; this will become important in what

\footnotetext{
${ }^{1}$ The dot $\cdot$ denotes inner product, and $\otimes$ is the tensor or outer product. Thus, in components, $\left(F^{\prime \prime}(u) \cdot v \otimes w\right)_{i}=\left(\partial^{2} F_{i} / \partial u_{j} \partial u_{k}\right) v_{j} w_{k}$, etc.
}

follows with time-dependent $w$. In our SWE illustration, the averaging operator picks up the resonant gravity wave triads and the vortical modes (the latter are always resonant on the fast timescale considered here); in the higher-order approximations considered below, we find resonances involving more than three modes. The integrating operator, on the other hand, acts only on the nonresonant terms in its operand.

Returning to our problem, at $O(\varepsilon)$ we find

$$
\begin{aligned}
\frac{d v^{1}}{d s} & =F\left(v^{0}, s\right)=F\left(v_{0}, s\right) \\
& \Rightarrow v^{1}(s)=v_{0}^{1}+\int_{0}^{s} F\left(v_{0}, r\right) d r .
\end{aligned}
$$

With the notation introduced above, the integral can be written as a sum of resonant and nonresonant parts,

$$
v^{1}(s)=v_{0}^{1}+s \overline{F\left(v_{0}\right)}+\underline{F\left(v_{0}, s\right)}-\underline{F\left(v_{0}, 0\right)} .
$$

In what follows we shall set the integration constants $v_{0}^{n}=0$ for $n \geq 1$. This choice implies that $v_{0}=v(0)$ $=u_{0}$; it also makes possible the identification in the next section of the slaving manifold as the invariant renormalization slow manifold. Other choices for $v_{0}^{n}$ are possible; for example, taking $v_{0}^{1}=F\left(v_{0}, 0\right)$ to cancel the constant term in (2.10) and similarly at higher orders corresponds to computing the normal form (Bruno 1989) in averaging-type approaches (Bogolyubov and Mitropol'skii 1958).

With $v_{0}^{1}=0$, our unrenormalized approximate solution to $O(\varepsilon)$ is then given by

$$
\begin{aligned}
\hat{v}^{1}(s): & =v^{0}(s)+\varepsilon v^{1}(s) \\
& =v_{0}+\varepsilon s \overline{F\left(v_{0}\right)}+\varepsilon \underline{F\left(v_{0}, s\right)}-\varepsilon \underline{F\left(v_{0}, 0\right)} .
\end{aligned}
$$

The secular term $\varepsilon s \overline{F\left(v_{0}\right)}$ can be removed by replacing the constant $v_{0}$ by a slowly time-varying variable $\bar{v}(s)$ in the following manner (Chen et al. 1996; Ziane 2000). Let $\bar{v}(s)$ be the solution of

$$
\frac{d \bar{v}}{d s}=\varepsilon \overline{F(\bar{v})}, \quad \bar{v}(0)=v_{0} ;
$$

note that $\bar{v}$ is the renormalized variable-the bar here does not denote averaging. It follows that $\bar{v}(s)=v_{0}+$ $\varepsilon s \overline{F(\bar{v}(s))}+O\left(\varepsilon^{2}\right)=v_{0}+\varepsilon s \overline{F\left(v_{0}\right)}+O\left(\varepsilon^{2}\right)$, so from (2.11) we obtain our $O(\varepsilon)$ renormalized solution

$$
\tilde{v}^{1}(s)=\bar{v}(s)+\varepsilon[\underline{F(\bar{v}(s), s)}-\underline{F(\bar{v}(s), 0)}] .
$$

Our final approximate solution to $O(\varepsilon)$ is then given by the first-order renormalized system (2.12) and its relation (2.13) to the original variable $v$.

The renormalized system (2.12) can therefore be thought of as a slow evolution equation for the amplitudes of all the variables in the system, with the alge- 
braic equation (2.13) accounting for all the fast linear oscillations that may be present.

Returning to (2.3)-(2.4), at the next order, we find (with $v_{0}^{2}=0$ )

$$
\begin{aligned}
v^{2}(s)= & \int_{0}^{s} F^{\prime}\left(v^{0}(r), r\right) \cdot v^{1}(r) d r \\
= & \frac{1}{2} s^{2} \overline{F^{\prime}\left(v_{0}\right)} \cdot \overline{F\left(v_{0}\right)}+s \underline{F^{\prime}\left(v_{0}, s\right)} \cdot \overline{F\left(v_{0}\right)} \\
& -\underline{\underline{F^{\prime}\left(v_{0}, s\right)}} \cdot \overline{F\left(v_{0}\right)}+\underline{\underline{F^{\prime}\left(v_{0}, 0\right)}} \cdot \overline{F\left(v_{0}\right)} \\
& +s \overline{F^{\prime}\left(v_{0}\right) \cdot \underline{F\left(v_{0}\right)}}+\underline{F^{\prime}\left(v_{0}, s\right)} \cdot \underline{F\left(v_{0}, s\right)} \\
& -\underline{F^{\prime}\left(v_{0}, 0\right)} \cdot \underline{F\left(v_{0}, 0\right)} \\
& -s \overline{F^{\prime}\left(v_{0}\right)} \cdot \underline{F\left(v_{0}, 0\right)}-\underline{F^{\prime}\left(v_{0}, s\right)} \cdot \underline{F\left(v_{0}, 0\right)} \\
& +\underline{F^{\prime}\left(v_{0}, 0\right)} \cdot \underline{F\left(v_{0}, 0\right)}
\end{aligned}
$$

after using (2.10) with $v_{0}^{1}=0$. A word on the notation: the integrating operator applied on $F^{\prime}\left(v_{0}, s\right) \cdot F\left(v_{0}, 0\right)$ gives $F^{\prime}\left(v_{0}, s\right) \cdot F\left(v_{0}, 0\right)$; when the latter is evaluated at

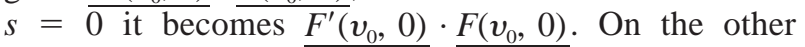
hand, the integrating operator applied on $F^{\prime}\left(v_{0}\right.$, $s) \cdot \underline{F\left(v_{0}, s\right)}$ gives $F^{\prime}\left(v_{0}, s\right) \cdot \underline{F\left(v_{0}, s\right)}$; the latter becomes $F^{\prime}\left(v_{0}, 0\right) \cdot F\left(v_{0}, 0\right)$ when evaluated at $s=0$.

We now proceed to remove the secular terms in $\hat{v}^{2}:=$ $v^{0}+\varepsilon v^{1}+\varepsilon^{2} v^{2}$ by renormalization. To wit, we replace $v_{0}=\bar{v}(0)$ by the Taylor series of $\bar{v}(s)$ around $s$ :

$$
v_{0}=\bar{v}(s)-s \frac{d \bar{v}}{d s}(s)+\frac{s^{2}}{2} \frac{d^{2} \bar{v}}{d s^{2}}(s)-\cdots,
$$

where $\bar{v}(s)$ satisfies the second-order renormalized equation

$$
\frac{d \bar{v}}{d s}=\varepsilon W_{1}(\bar{v})+\varepsilon^{2} W_{2}(\bar{v}), \quad \bar{v}(0)=v_{0} .
$$

From the previous order, we have found that $W_{1}(\bar{v})=$ $\overline{F(\bar{v})}$, so $(2.15)$ becomes

$$
\begin{aligned}
v_{0}= & \bar{v}(s)-\varepsilon s \overline{F(\bar{v})}+\frac{1}{2} \varepsilon^{2} s^{2} \overline{F^{\prime}(\bar{v})} \cdot \overline{F(\bar{v})} \\
& -\varepsilon^{2} s W_{2}(\bar{v})+O\left(\varepsilon^{3}\right),
\end{aligned}
$$

where $W_{2}$ will be determined shortly. (Note that taking the $\bar{v}$ derivative of $F$ commutes with both the averaging and integrating operators.) Substituting this expression into

$$
\begin{aligned}
\hat{v}^{2}= & v_{0}+\varepsilon\left[s \overline{F\left(v_{0}\right)}+\underline{F\left(v_{0}, s\right)}-\underline{F\left(v_{0}, 0\right)}\right] \\
& +\varepsilon^{2}\left[\frac{1}{2} s^{2} \overline{F^{\prime}\left(v_{0}\right)} \cdot \overline{F\left(v_{0}\right)}+s \underline{F^{\prime}\left(v_{0}, s\right)} \cdot \overline{F\left(v_{0}\right)}\right.
\end{aligned}
$$

$$
\begin{aligned}
& -\underline{\underline{F^{\prime}\left(v_{0}, s\right)}} \cdot \overline{F\left(v_{0}\right)}+\underline{\underline{F^{\prime}\left(v_{0}, 0\right)}} \cdot \overline{F\left(v_{0}\right)} \\
& +s \overline{F^{\prime}\left(v_{0}\right) \cdot \underline{F\left(v_{0}\right)}}+\underline{F^{\prime}\left(v_{0}, s\right) \cdot \underline{F\left(v_{0}, s\right)}} \\
& -\underline{F^{\prime}\left(v_{0}, 0\right) \cdot \underline{F\left(v_{0}, 0\right)}} \\
& -s \overline{F^{\prime}\left(v_{0}\right)} \cdot \underline{F\left(v_{0}, 0\right)}-\underline{F^{\prime}\left(v_{0}, s\right)} \cdot \underline{F\left(v_{0}, 0\right)} \\
& \left.+\underline{F^{\prime}\left(v_{0}, 0\right)} \cdot \underline{F\left(v_{0}, 0\right)}\right],
\end{aligned}
$$

and dropping terms of $O\left(\varepsilon^{3}\right)$ or higher, we find

$$
\begin{aligned}
& \hat{v}^{2}=\bar{v}(s)-\varepsilon s \overline{F(\bar{v})}+\frac{1}{2} \varepsilon^{2} s^{2} \overline{F^{\prime}(\bar{v})} \cdot \overline{F(\bar{v})}-\varepsilon^{2} s W_{2}(\bar{v}) \\
& +\varepsilon s \overline{F(\bar{v})}-\varepsilon^{2} s^{2} \overline{F^{\prime}(\bar{v})} \cdot \overline{F(\bar{v})}+\varepsilon \underline{F(\bar{v}, s)} \\
& -\varepsilon^{2} s \underline{F^{\prime}(\bar{v}, s)} \cdot \overline{F(\bar{v})}-\varepsilon \underline{F(\bar{v}, 0)} \\
& +\varepsilon^{2} s \underline{F^{\prime}(\bar{v}, 0)} \cdot \overline{F(\bar{v})} \\
& +\varepsilon^{2}\left[\frac{1}{2} s^{2} \overline{F^{\prime}(\bar{v})} \cdot \overline{F(\bar{v})}+s \underline{F^{\prime}(\bar{v}, s)} \cdot \overline{F(\bar{v})}\right. \\
& -\underline{\underline{F^{\prime}(\bar{v}, s)}} \cdot \overline{F(\bar{v})}+\underline{\underline{F^{\prime}(\bar{v}, 0)}} \cdot \overline{F(\bar{v})} \\
& +s \overline{F^{\prime}(\bar{v}) \cdot \underline{F(\bar{v})}}+F^{\prime}(\bar{v}, s) \cdot \underline{F(\bar{v}, s)} \\
& -F^{\prime}(\bar{v}, 0) \cdot \underline{F(\bar{v}, 0)} \\
& -s \overline{F^{\prime}(\bar{v})} \cdot \underline{F(\bar{v}, 0)}-\underline{F^{\prime}(\bar{v}, s)} \cdot \underline{F(\bar{v}, 0)} \\
& \left.+\underline{F^{\prime}(\bar{v}, 0)} \cdot \underline{F(\bar{v}, 0)}\right]+O\left(\varepsilon^{3}\right) .
\end{aligned}
$$

The $O(\varepsilon)$ secular terms cancel by construction, while the $O\left(\varepsilon^{2}\right)$ secular terms can be removed by choosing

$$
\begin{aligned}
W_{2}(\bar{v})= & \overline{F^{\prime}(\bar{v}) \cdot \underline{F(\bar{v})}}-\overline{F^{\prime}(\bar{v})} \cdot \underline{F(\bar{v}, 0)} \\
& +\underline{F^{\prime}(\bar{v}, 0)} \cdot \overline{F(\bar{v})},
\end{aligned}
$$

leaving us with the renormalized solution

$$
\begin{aligned}
& \tilde{v}^{2}(s)=\bar{v}(s)+\varepsilon[F(\bar{v}, s)-F(\bar{v}, 0)] \\
& +\varepsilon^{2}\left[F^{\prime}(\bar{v}, s) \cdot F(\bar{v}, s)-F^{\prime}(\bar{v}, s) \cdot \overline{F(\bar{v})}\right. \\
& -\underline{F^{\prime}(\bar{v}, s)} \cdot \underline{F(\bar{v}, 0)} \\
& -F^{\prime}(\bar{v}, 0) \cdot \underline{F(\bar{v}, 0)}+\underline{F^{\prime}(\bar{v}, 0)} \cdot \overline{F(\bar{v})} \\
& \left.+F^{\prime}(\bar{v}, 0) \cdot F(\bar{v}, 0)\right] .
\end{aligned}
$$

To this order, the approximate solution consists of (2.19) along with the evolution (renormalized) equation for $\bar{v}(s)$,

$$
\begin{gathered}
\frac{d \bar{v}}{d s}=\varepsilon \overline{F(\bar{v})}+\varepsilon^{2}\left[\overline{F^{\prime}(\bar{v}) \cdot \underline{F(\bar{v})}}-\overline{F^{\prime}(\bar{v})} \cdot \underline{F(\bar{v}, 0)}\right. \\
\left.+\underline{F^{\prime}(\bar{v}, 0)} \cdot \overline{F(\bar{v})}\right]
\end{gathered}
$$

with $\bar{v}(0)=v_{0}$ 
We note that the quadratic term $s^{2} \overline{F^{\prime}} \cdot \bar{F}$ and the oscillating secular term $s F^{\prime} \cdot \bar{F}$, which appear at $O\left(\varepsilon^{2}\right)$, all cancel due to our $O(\overline{\varepsilon)}$ renormalization of the linear secular term $s \bar{F}$. With some more computation (which we do not show here), it can be verified that the same holds at the next order. Subject to this renormalizability condition, we believe that the procedure can be carried on (at least formally for PDEs-see below) to higher orders to obtain better approximations, although the amount of computation increases very quickly.

In most applications, what one would be interested in is the renormalized evolution equation, the analogue of (2.20), since this may yield information about weak higher-order interactions that become important over long timescales. Less likely to be useful is the higherorder analogue of (2.19), which would only give a small algebraic correction that does not grow with time. In this way, the renormalization procedure can be regarded as a method to separate long-term evolution from shortterm rapid bounded oscillations.

For finite-dimensional systems, it can be shown rigorously (Temam and Wirosoetisno 2002) that our renormalized approximate solutions $\tilde{v}^{1}(s), \widetilde{v}^{2}(s)$ are uniformly valid over times $s \sim \varepsilon^{-1}$ up to the order specified, as long as the solution of the evolution equation [the analogue of (2.20) to the appropriate order] exists. More precisely, the exact solution $v(s)$ of (2.2) is approximated by $\tilde{v}^{n}(s)$ in the following sense. For fixed constants $C_{i}$ and $T_{i}$, which depend on the initial conditions but not on $\varepsilon$, as $\varepsilon \rightarrow 0$ :

$$
\begin{aligned}
& \left|v(s)-\widetilde{v}^{n}(s)\right| \leq C_{1} \varepsilon^{n} \quad \text { for } s \leq T_{1} / \varepsilon, \\
& \left|v(s)-\tilde{\boldsymbol{v}}^{n}(s)\right| \leq C_{2} \varepsilon^{n-1} \quad \text { for } s \leq-T_{2} \log \varepsilon / \varepsilon .
\end{aligned}
$$

In the slow time $t$, (i) holds for $t \leq T_{1}$, that is, for several (order 1) eddy turnaround times, and (ii) holds for a factor of $\log \varepsilon$ longer.

For infinite-dimensional systems, such as the partial differential equations of GFD, further issues have to be addressed. One is the small denominator problem ${ }^{2}$ mentioned in section 4. Ensuring that the solution of the renormalized evolution equation remains regular over the timescales in (i) and (ii) of (2.21) above also becomes more difficult. These problems are nontrivial in most cases (and may not be possible beyond a certain order $n$ ), but since the issue of regularity is well beyond the scope of this paper we shall not discuss it here.

Finally, we note that the slow evolution equation (2.20) and its relation to the original variables (2.19) obtained above using renormalization can also be obtained using the averaging method introduced by Kry-

\footnotetext{
${ }^{2}$ In the finite-dimensional case, small denominators do not cause problems as long as one works with a finite order of the expansion. They do cause nontrivial difficulties when one goes to all orders [as in (Kolmogorov-Arnol'd-Moser) KAM-type theorems] or when the order is taken as a function of $\varepsilon$ (as in Nekhoroshev-type theorems).
}

lov, Bogolyubov, and Mitropol'skii. In fact, one can verify directly that the two methods are equivalent for the first few orders - they are probably equivalent to all orders. We have adopted the renormalization approach here since the idea also applies to the removal of secularity in the slaving solution of Warn et al. (1995) discussed in the next section.

\section{Connection with the slow manifold}

There is a natural and close connection between the renormalized solution obtained in the preceding section and the so-called slow manifold, defined by the slaving procedure (e.g., Warn et al. 1995).

To show this, we rewrite the system (2.1) on the slow time $t:=\varepsilon s$ by a slight abuse of notation $[u$ is either $u(s)$ or $u(t)$ depending on context]:

$$
\frac{d u}{d t}+\frac{L}{\varepsilon} u=\mathcal{F}(u)
$$

If we expand $u(t)$ in powers of $\varepsilon$ as before, we find that at leading order

$$
L u^{0}=0 .
$$

This suggests separating $u$ into a "slow" component $y$ that belongs to ker $L$ and its $L$-orthogonal "fast" complement $x$. We thus write $u=(x, y)$. With these variables, (3.1) can be written as

$$
\begin{aligned}
\frac{d x}{d t}+\frac{L_{x}}{\varepsilon} x & =x(x, y) \\
\frac{d y}{d t} & =y(x, y),
\end{aligned}
$$

where $L_{x}$ is the restriction of $L$ to its resolvent (and is thus nonsingular), and $x$ and $y$ are the $x$ and $y$ components of $\mathcal{F}$, respectively.

We can now expand both $x(t)$ and $y(t)$ in powers of $\varepsilon$ and solve for them order by order as before. This is the slow time expansion alluded to earlier, and is the basis for the systematic derivation of the quasigeostrophic model (cf. Pedlosky 1987, section 3.12). When we do this, we find that $x^{n}$ can always be determined from previous iterations:

$$
x^{0}=0, \quad L_{x} x^{1}=x\left(0, y^{0}\right), \text { etc. }
$$

So (our approximation to) the fast variable $x$ has no independent evolution equation of its own; in other words, it is slaved. Hence the term reductive perturbation theory [note that this is not the sense in which it is used by Chen et al. (1996)]. In the more mathematical literature (e.g., Schochet 1994; Embid and Majda 1996), this case is often referred to as solving a problem with well-prepared initial data.

Put differently, this expansion cannot give us any generic solutions, but only those living in a (slow) solution manifold $\mathcal{S}_{\text {slav }}$ [see (3.8) below]. This restriction is understood for the remainder of this section. 
As pointed out in Warn et al. (1995), however, this program soon runs into a difficulty: at $O(\varepsilon)$ we find

$$
\frac{d y^{1}}{d t}=\mathcal{Y}_{y}\left(0, y^{0}\right) \cdot y^{1}+\mathcal{Y}_{x}\left(0, y^{0}\right) \cdot x^{1} .
$$

Apart from the second term on the right-hand side, this equation describes the evolution of a Lyapunov vector of the system

$$
\frac{d y^{0}}{d t}=\mathcal{Y}\left(0, y^{0}\right)
$$

implying that if this latter system possesses a positive Lyapunov exponent then $y^{1}$ will grow exponentially in time. This is an undesirable situation, since in most applications one would like an approximate system that is well behaved, even when outside the timescale of validity of the original approximation.

This blow up can be handled in much the same way as the secular solutions we encountered earlier-by renormalization. Since $y$ is the only prognostic variable ( $x$ is slaved to $y$ ), we only need to renormalize $y$. Here the procedure is quite simple: to $O(\varepsilon)$, define $\overline{\bar{y}}:=y^{0}$ $+\varepsilon y^{1}$; we then find, using (3.4), (3.5) and (3.6), and undoing the Taylor expansion, that the time evolution of $\overline{\bar{y}}(t)$ is given by

$$
\frac{d \overline{\bar{y}}}{d t}=\mathcal{Y}\left(\varepsilon L_{x}^{-1} x(0, \overline{\bar{y}}), \overline{\bar{y}}\right)+O\left(\varepsilon^{2}\right),
$$

the $O(\varepsilon)$ truncation of which no longer blows up. It can easily be verified that the same procedure works at higher orders.

This approach is of course equivalent to not expanding the slow variable $y$, as proposed in the slaving approach of Warn et al. (1995). We can thus do away with the renormalized variable $\overline{\bar{y}}$ and simply write $y$ in its place.

Since we know that $x$ is slaved to $y$ on $\mathcal{S}_{\text {slav }}$, we write it as a function of $y$, namely,

$$
\begin{aligned}
x & =U(y ; \varepsilon) \\
& =U_{0}(y)+\varepsilon U_{1}(y)+\varepsilon^{2} U_{2}(y)+\cdots .
\end{aligned}
$$

Our solution manifold is then $\mathcal{S}_{\text {slav }}:=\{(x, y) \mid x=$ $U(y ; \varepsilon)\}$. Using (3.3), we find that the slaving relation $U(y ; \varepsilon)$ is determined implicitly by the so-called superbalance equation (Lorenz 1980)

$$
\begin{aligned}
& U^{\prime}(y ; \varepsilon) \mathcal{Y}(U(y ; \varepsilon), y)+\frac{L_{x}}{\varepsilon} U(y ; \varepsilon) \\
& \quad=X(U(y ; \varepsilon), y) .
\end{aligned}
$$

At the three lowest orders, the superbalance equation yields

$$
\begin{aligned}
& U_{0}(y)=0 \\
& U_{1}(y)=L_{x}^{-1} X(0, y) \\
& U_{2}(y)=L_{x}^{-1}\left[X_{x}(0, y) L_{x}^{-1} \chi(0, y)\right. \\
& \text { - } \left.L_{x}^{-1} X_{y}(0, y) \mathcal{Y}(0, y)\right] \text {. }
\end{aligned}
$$

As noted earlier, this procedure is not able to capture the general solutions such as those obtained in the previous section using the renormalization method; rather, it only gives us solutions lying on a special manifold $S_{\text {slav }}$. It is moreover clear that the slaving construction rules out rapid oscillations that are generically present in the general solution, so $\mathcal{S}_{\text {slav }}$ is in a sense slow. The rest of this section is devoted to making this statement more precise, which we do by finding a set of slow renormalized solutions and then showing that it corresponds precisely to $\mathcal{S}_{\text {slav }}$ defined above.

To do this we shift gears and return to our $O\left(\varepsilon^{2}\right)$ renormalized system (2.19)-(2.20). We look for the condition such that our original variable $u(s)$ is free of fast oscillations. Denoting the right-hand side of (2.19) by $R(\bar{v}, s)$ so that the approximate solution for $u(s)$ is given by $\tilde{u}^{2}(s)=e^{-L s} \tilde{\boldsymbol{v}}^{2}(s)=e^{-L s} R(\bar{v}, s)$, we demand that $\tilde{u}^{2}(s)$ is completely slow or, in other words, that

$$
\frac{\partial}{\partial s}\left[e^{-L s} R(\bar{v}, s)\right]=0 .
$$

Introducing the notations $\tilde{u}=(\tilde{x}, \tilde{y})$ and $F=(X, Y)$, we find from (2.19) that at $O(1)$,

$$
\begin{aligned}
& \tilde{x}^{0}(s)=e^{-L_{x} s} \bar{x}(s) \\
& \tilde{y}^{0}(s)=\bar{y}(s),
\end{aligned}
$$

where $\bar{v}=(\bar{x}, \bar{y})$. [Note that the $y$ components of $u$ and $v$ are the same, since $e^{L s}(0, y)=(0, y)$.] Now (3.12a) implies that, for $\tilde{u}^{0}(s)$ to be slow, one must have $\bar{x} \sim$ $O(\varepsilon)$. Writing $\bar{x}=\varepsilon \Psi_{1}$, up to $O(\varepsilon)$, with $\Psi_{1}$ to be determined, (2.19) gives

$$
\begin{aligned}
\tilde{x}^{-1}(s)= & \varepsilon e^{-L_{x} s} \Psi_{1}+\varepsilon e^{-L_{x} s} \underline{X(\bar{x}, \bar{y}, s)} \\
& -\varepsilon e^{-L_{x} s} \underline{X(\bar{x}, \bar{y}, 0)} \\
= & \varepsilon e^{-L_{x} s} \Psi_{1}+\varepsilon e^{-L_{x} s} \underline{X(0, \bar{y}, s)} \\
& -\varepsilon e^{-L_{x} s} \underline{X(0, \bar{y}, 0)}+O\left(\varepsilon^{2}\right) .
\end{aligned}
$$

To proceed further, we need to revisit the definition of $\underline{X(\bar{x}, \bar{y}, s)}$. At $\bar{x}=0,(2.8)$ gives

$$
\begin{aligned}
& \frac{X(0, \bar{y}, s)}{\quad}-\underline{X(0, \bar{y}, 0)} \\
& \quad=\int_{0}^{s} e^{L_{x} r} X(0, \bar{y}) d r \\
& \quad=L_{x}^{-1} e^{L_{x} s} X(0, \bar{y})-L_{x}^{-1} X(0, \bar{y}),
\end{aligned}
$$

noting that the integrand only contains nonresonant parts ( $\bar{y}$ is held fixed in the integration). It follows that

$$
\begin{aligned}
\underline{X(0, \bar{y}, s)} & =e^{L_{x} s} \underline{X(0, \bar{y}, 0)} \\
& =e^{L_{x} s} L_{x}^{-1} X(0, \bar{y}) .
\end{aligned}
$$

Substituting the first line of the last expression into (3.13), we find 


$$
\begin{aligned}
\tilde{x}^{1}(s)= & \varepsilon e^{-L_{x} s} \Psi_{1}+\varepsilon \underline{X(0, \bar{y}, 0)} \\
& -\varepsilon e^{-L_{x} s} \underline{X(0, \bar{y}, 0)},
\end{aligned}
$$

which can be made completely slow to this order provided we take

$$
\Psi_{1}=\underline{X(0, \bar{y}, 0)}=L_{x}^{-1} X(0, \bar{y}) .
$$

[It may be noted that this is just the $O(\varepsilon)$ slaving relation (3.10b), with $\bar{y}$ in place of $y$.] Hence, $\tilde{x}^{1}=\bar{x}$ to this order.

We now consider $\tilde{x}^{2}(s)$, which, using the earlier cancellations in $\tilde{x}^{1}(s)$ and writing $\bar{x}=\varepsilon X(0, \bar{y}, 0)+\varepsilon^{2} \Psi_{2}$ $+\cdots$, is given by [cf. (2.19)]

$$
\begin{aligned}
& \tilde{x}^{2}(s)=\varepsilon \underline{X(0, \bar{y}, 0)}+\varepsilon^{2} e^{-L_{x} s}\left[\Psi_{2}+\underline{X_{x}(0, \bar{y}, s)} \cdot \underline{X(0, \bar{y}, 0)}-\underline{X_{x}(0, \bar{y}, 0)} \cdot \underline{X(0, \bar{y}, 0)}\right. \\
& +X_{x}(0, \bar{y}, s) \cdot \underline{X(0, \bar{y}, s)}-\underline{X_{x}(0, \bar{y}, s)} \cdot \overline{X(0, \bar{y})}-\underline{X_{x}(0, \bar{y}, s)} \cdot \underline{X(0, \bar{y}, 0)} \\
& +\underline{X_{y}(0, \bar{y}, s) \cdot \underline{Y(0, \bar{y}, s)}}-\underline{X_{y}(0, \bar{y}, s)} \cdot \overline{Y(0, \bar{y})}-\underline{X_{y}(0, \bar{y}, s)} \cdot \underline{Y(0, \bar{y}, 0)} \\
& -\underline{X_{x}(0, \bar{y}, 0) \cdot \underline{X(0, \bar{y}, 0)}}+\underline{\underline{X_{x}(0, \bar{y}, 0)}} \cdot \overline{X(0, \bar{y})}+\underline{X_{x}(0, \bar{y}, 0)} \cdot \underline{X(0, \bar{y}, 0)}
\end{aligned}
$$

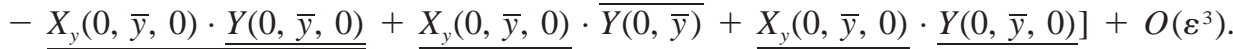

A few more identities analogous to (3.14) are needed for the next step:

$$
\begin{aligned}
& Y(0, \bar{y}, s)=0, \quad \overline{X(0, \bar{y})}=0, \\
& \underline{X_{x}(0, \bar{y}, s) \cdot \underline{X(0, \bar{y}, s)}} \\
& =e^{L_{x} s} X_{x}(0, \bar{y}, 0) \cdot X(0, \bar{y}, 0), \\
& \underline{\underline{X_{y}(0, \bar{y}, s)}} \cdot \overline{Y(0, \bar{y})} \\
& =e^{L_{x} s} X_{y}(0, \bar{y}, 0) \cdot \overline{Y(0, \bar{y})} .
\end{aligned}
$$

Effecting the resulting cancellations, and upon choosing

$$
\Psi_{2}=\frac{X_{x}(0, \bar{y}, 0) \cdot \underline{X(0, \bar{y}, 0)}}{-\underline{\underline{X_{y}(0, \bar{y}, 0)} \cdot \overline{Y(0, \bar{y})}}}
$$

we find that $\tilde{x}^{2}(s)$ has become completely slow to this order. Expressing $(X, Y)$ in terms of $(X, Y)$ [cf. (3.14)], this recovers the $O\left(\varepsilon^{2}\right)$ slaving relation (3.10c), with $\bar{y}$ in place of $y$. As $\tilde{x}^{2}(s)$ is completely slow, $\tilde{x}^{2}=\bar{x}$ to this order. In addition, since no constraint has been imposed on $\tilde{y}$, one obviously has $\tilde{y}=\bar{y}$ exactly.

After much computation (of which we shall spare the reader), it can be verified that imposing slowness on the $O\left(\varepsilon^{3}\right)$ renormalized solution also yields the slaving relation at that order. We conclude then that, to $O\left(\varepsilon^{3}\right)$, the special renormalization solutions that are free of fast oscillations lie on the slow manifold obtained using the slaving procedure. In other words, the set of slow renormalized solutions, which we will denote by $\mathcal{S}_{\text {rg }}$, coincides with the slaving manifold $\mathcal{S}_{\text {slav }}$. We will henceforth write $\mathcal{S}$ for both $\mathcal{S}_{\text {rg }}$ and $\mathcal{S}_{\text {slav }}$.

Several further remarks are in order.

First, the slow manifold $S$ turns out to be the fixed point of the transformation that gives the original $u$ variable from the renormalized variable $\bar{v}$; that is, it solves

$$
\bar{v}=e^{-L s} R(\bar{v}, s) \quad \text { for } \bar{v} \text { on } \mathcal{S}
$$

[recall that $R(\bar{v}, s)$ is the right-hand side of (2.19)]. This can be seen perhaps more directly by integrating (3.11), remembering that at $s=0, u=\bar{v}$. This manifold has a dimension that is equal to $\operatorname{dim} \operatorname{ker} L$, and it reduces to a point $\{0\}$ under our hypothesis $\mathcal{F}(0)=0$ when $v$ consists only of fast variables.

Second and more importantly, our renormalization solution allows us to (i) explicitly verify the invariance of $\mathcal{S}$ to the order of approximation considered, and (ii) investigate the behavior of the solution in the neighborhood of $\mathcal{S}$. We shall do this in the renormalized variables $\bar{v}=(\bar{x}, \bar{y})$. Writing $\Psi(\bar{y}, \varepsilon):=\varepsilon \Psi_{1}+$ $\varepsilon^{2} \Psi_{2}+\cdots$, let $\delta:=\bar{x}-\Psi(\bar{y} ; \varepsilon)$ denote the departure of the solution from $\mathcal{S}$. Returning to (2.20), we compute

$$
\begin{aligned}
& \frac{d \delta}{d s}=\varepsilon \overline{X(\bar{x}, \bar{y})} \\
& +\varepsilon^{2}\left[\overline{X_{x}(\bar{x}, \bar{y}) \cdot \underline{X(\bar{x}, \bar{y})}}-\overline{X_{x}(\bar{x}, \bar{y})} \cdot \underline{X(\bar{x}, \bar{y}, 0)}\right. \\
& +X_{x}(\bar{x}, \bar{y}, 0) \cdot \overline{X(\bar{x}, \bar{y})} \\
& +\overline{X_{y}(\bar{x}, \bar{y}) \cdot \underline{Y(\bar{x}, \bar{y})}} \\
& -\overline{X_{y}(\bar{x}, \bar{y})} \cdot Y(\bar{x}, \bar{y}, 0) \\
& \left.+X_{y}(\bar{x}, \bar{y}, 0) \cdot \overline{Y(\bar{x}, \bar{y})}\right] \\
& -\varepsilon \Psi^{\prime}(\bar{y} ; \varepsilon) \cdot \overline{Y(\bar{x}, \bar{y})}+O\left(\varepsilon^{3}\right),
\end{aligned}
$$

noting that $\Psi^{\prime}(\bar{y} ; \varepsilon) \sim O(\varepsilon)$. We consider the dynamics for small values of $\delta$. Expanding all $\bar{x}$ on the rhs around 0 , and upon using (3.18) and $\overline{X_{x}(0, \bar{y}) \cdot X(0, \bar{y})}=0$, we indeed find that $S$ is invariant to the stated order: 


$$
\begin{aligned}
& \frac{d \delta}{d s}=\varepsilon \overline{X_{x}(0, \bar{y})} \cdot \delta+\varepsilon^{2}\left\{\overline{X_{x x}(0, \bar{y}) \cdot \underline{X(0, \bar{y})}} \otimes \delta+\overline{X_{x}(0, \bar{y}) \cdot \underline{X_{x}(0, \bar{y})}} \cdot \delta-\overline{X_{x}(0, \bar{y})} \cdot \underline{X_{x}(0, \bar{y}, 0)} \cdot \delta\right. \\
& -\overline{X_{x x}(0, \bar{y})} \cdot X(0, \bar{y}, 0) \otimes \delta+\underline{X_{x}(0, \bar{y}, 0)} \cdot \overline{X_{x}(0, \bar{y})} \cdot \delta+\overline{X_{y}(0, \bar{y}) \cdot \underline{Y_{x}(0, \bar{y})}} \cdot \delta \\
& \left.+X_{y x}(0, \bar{y}) \cdot \overline{Y(0, \bar{y})} \otimes \delta\right\}+O\left(\varepsilon^{3}, \delta^{2}\right) .
\end{aligned}
$$

This invariance property (which also holds to the next order) cannot be obtained from the slaving procedure itself, so our present approach can be seen as providing a justification for the former (cf. also the appendix of Wirosoetisno and Shepherd 2000).

Finally, the reader may ask, what about the $y$ equations that were never used in computing $S$ ? At $O(\varepsilon)$, the property $Y(0, \bar{y}, s)=0$ gives $0=0$, and imposing slowness at the next order gives $\bar{x}=\varepsilon X(0, \bar{y}, 0)$. It turns out that the $y$ equation does not yield any new information, and it lags behind the $x$ equation used above by one order.

At leading order, the connection between the fast and slow time expansions discussed in this section is quite clear and no doubt has long been known to many people. At higher orders, however, there is an ambiguity in the fast time expansion arising from the choice of integration constants [cf. the discussion following (2.10)]—only the choice used here provides the connection.

\section{Renormalized equations for the weak-wave model}

In this section we provide an explicit worked example of the above formalism using a simplified model of the shallow-water equations valid for weak imbalance, the so-called weak-wave model of Nore and Shepherd (1997). We note that the physical conclusions obtained here may be at variance with those obtained with other models (e.g., the shallow-water equations). The development in this section is to be regarded as formal. We shall avoid technical issues such as whether the sums over wavenumbers in (4.11) and (4.16) and their higher-order analogues actually converge (to prove convergence would require estimates of small denominators; see further comments below), and more difficult issues such as whether the solution of the renormalized evolution equations stays bounded and smooth, etc.

Taking the quasigeostrophic potential vorticity $\tilde{q}$, divergence $\Delta$, and disturbance height $\eta$ as the dependent variables, the governing equations of the model are as follows:

$$
\tilde{q}_{t}=-\partial(\psi, \tilde{q})
$$

$$
\begin{aligned}
\Delta_{t} & =\frac{1}{\varepsilon}\left(\frac{B^{2}-\nabla^{2}}{1+B^{2}}\right) \eta+\frac{1}{\varepsilon} b \tilde{q} \\
\eta_{t} & =-\frac{1}{\varepsilon} \Delta,
\end{aligned}
$$

with $\tilde{q}=: \nabla^{2} \psi-b \eta$. Here $B:=f L / \sqrt{g H}$ is the rotational Froude number, $b:=B / \sqrt{1+B^{2}}$, and $\varepsilon:=$ Ro $\cdot b$ is the timescale separation parameter (Ro is the Rossby number). For more details on the derivation of the model, the reader is referred to Nore and Shepherd (1997).

For the development below, we need to write the system in terms of fast and slow variables. Of the original variables $(\tilde{q}, \Delta, \eta), \eta$ is not completely fast at leading order (as can be seen from the equations of motion), so we shall replace it with its ageostrophic part,

$$
h:=\eta+b\left(\frac{B^{2}-\nabla^{2}}{1+B^{2}}\right)^{-1} \tilde{q} .
$$

Using $(\tilde{q}, \Delta, h)$ as our new set of dependent variables, the governing equations (4.1) read

$$
\begin{aligned}
& \tilde{q}_{t}=-\partial(\psi, \tilde{q}) \\
& \Delta_{t}=\frac{1}{\varepsilon}\left(\frac{B^{2}-\nabla^{2}}{1+B^{2}}\right) h \\
& h_{t}=-\frac{1}{\varepsilon} \Delta-b\left(\frac{B^{2}-\nabla^{2}}{1+B^{2}}\right)^{-1} \partial(\psi, \tilde{q}),
\end{aligned}
$$

where now $\psi$ is to be computed from $\tilde{q}$ and $h$.

In this paper, we shall work in a two-dimensional periodic domain $x \in \Omega:=[0,2 \pi) \times[0,2 \pi a)$. To apply the above results to the model, it is most convenient to work in terms of Fourier modes. We write

$$
\begin{aligned}
\tilde{q}(x, t) & =\frac{1}{2 \pi \sqrt{a}} \sum_{k} q_{k}(t) e^{i k \cdot x} \text { and } \\
q_{k}(t) & =\frac{1}{2 \pi \sqrt{a}} \int \tilde{q}(x, t) e^{-i k \cdot x} d x,
\end{aligned}
$$

with similar expressions for $\Delta(x)$ and $h(x)$. We assume that all quantities have vanishing averages, so the $k=$ 0 components are identically zero [thus $h$ in (4.2) is unambiguously defined]. Here and henceforth, it is un- 
derstood that unless otherwise specified summations are taken over $k=\left(k_{1}, k_{2}\right) \in \mathbf{Z}^{2} \backslash\{0\}$, and integrations are taken over $\Omega$. The dot product is defined as $k \cdot x:=k_{1} x_{1}$ $+k_{2} x_{2} / a$.

The discrete version of the equations of motion (4.3) reads

$$
\begin{aligned}
& \dot{q}_{k}=\frac{\varepsilon}{2 \pi \sqrt{a}} \sum_{n}(k \times n) q_{k-n}\left(\gamma_{n} q_{n}+\frac{b}{n^{2 *}} h_{n}\right) \\
& \dot{\Delta}_{k}=\lambda_{k}^{2} h_{k} \\
& \dot{h}_{k}=-\Delta_{k}+\frac{\varepsilon b}{2 \pi \sqrt{a}} \lambda_{k}^{-2} \sum_{n}(k \times n) q_{k-n}\left(\gamma_{n} q_{n}+\frac{b}{n^{2 *}} h_{n}\right) .
\end{aligned}
$$

Here, an overdot denotes time $s$ derivative, $k \times n:=$ $\left(k_{1} n_{2}-k_{2} n_{1}\right) / a, n^{2 *}:=n_{1}^{2}+n_{2}^{2} / a^{2}$, and

$$
\lambda_{k}:=\sqrt{\frac{B^{2}+k^{2 *}}{B^{2}+1}} \quad \gamma_{k}:=\frac{1}{B^{2}+k^{2 *}} .
$$

To make the algebra more transparent, we diagonalize the fast part of (4.5) by introducing the variables

$$
\xi_{k}:=\frac{1}{2}\left(\lambda_{k}^{-1} \Delta_{k}+i h_{k}\right)
$$

$$
\zeta_{k}:=\frac{1}{2}\left(h_{k}+i \lambda_{k}^{-1} \Delta_{k}\right)
$$

In the variables $\left(q_{k}, \xi_{k}, \zeta_{k}\right),(4.5)$ becomes

$$
\begin{aligned}
\dot{q}_{k}= & \frac{\varepsilon}{2 \pi \sqrt{a}} \sum_{n}(k \times n) q_{k-n}\left[\gamma_{n} q_{n}+\frac{b}{n^{2 *}}\left(\zeta_{n}-i \xi_{n}\right)\right] \\
\dot{\xi}_{k}= & -i \lambda_{k} \xi_{k}+\varepsilon \frac{i b}{4 \pi \sqrt{a}} \lambda_{k}^{-2} \\
& \times \sum_{n}(k \times n) q_{k-n}\left[\gamma_{n} q_{n}+\frac{b}{n^{2 *}}\left(\zeta_{n}-i \xi_{n}\right)\right] \\
\dot{\zeta}_{k}= & i \lambda_{k} \zeta_{k}+\varepsilon \frac{b}{4 \pi \sqrt{a}} \lambda_{k}^{-2} \\
& \times \sum_{n}(k \times n) q_{k-n}\left[\gamma_{n} q_{n}+\frac{b}{n^{2 *}}\left(\zeta_{n}-i \xi_{n}\right)\right] .
\end{aligned}
$$

In the renormalization notation of the previous sections $u=\left(q_{k}, \xi_{k}, \zeta_{k}\right)$ and (4.8) represent (2.1). We have $v=\left(q_{k}, \xi_{k}^{\prime}, \zeta_{k}^{\prime}\right):=\left(q_{k}, e^{i \lambda_{k} s} \xi_{k}, e^{-i \lambda_{k} s} \zeta_{k}\right)$, and the analogue of (2.2) is

$$
\begin{aligned}
& \frac{d q_{k}}{d s}=\frac{\varepsilon}{2 \pi \sqrt{a}} \sum_{n}(k \times n) q_{k-n}\left[\gamma_{n} q_{n}+\frac{b}{n^{2 *}}\left(e^{i \lambda_{n} s} \zeta_{n}^{\prime}-i e^{-i \lambda_{n} s} \xi_{n}^{\prime}\right)\right] \\
& \frac{d \xi_{k}^{\prime}}{d s}=\varepsilon \frac{i b}{4 \pi \sqrt{a}} \lambda_{k}^{-2} e^{i \lambda_{k} s} \sum_{n}(k \times n) q_{k-n}\left[\gamma_{n} q_{n}+\frac{b}{n^{2 *}}\left(e^{i \lambda_{n} s} \zeta_{n}^{\prime}-i e^{-i \lambda_{n} s} \xi_{n}^{\prime}\right)\right] \\
& \frac{d \zeta_{k}^{\prime}}{d s}=\varepsilon \frac{b}{4 \pi \sqrt{a}} \lambda_{k}^{-2} e^{-i \lambda_{k} s} \sum_{n}(k \times n) q_{k-n}\left[\gamma_{n} q_{n}+\frac{b}{n^{2 *}}\left(e^{i \lambda_{n} s} \zeta_{n}^{\prime}-i e^{-i \lambda_{n} s} \xi_{n}^{\prime}\right)\right] .
\end{aligned}
$$

Ignoring the $k=0$ modes (which are zero by hypothesis), (4.6) implies that (i) $\lambda_{k}=\lambda_{n}$ if and only if $k^{2 *}=n^{2 *}$ and, (ii) $\lambda_{k}>0$ for any $k \neq 0$. Now the $O(\varepsilon)$ resonant terms can be read off (4.9), giving the evolution equations for the renormalized variables $\bar{v}=\left(\bar{q}_{k}, \bar{\xi}_{k}, \bar{\zeta}_{k}\right)$ [cf. (2.12)]:

$$
\begin{aligned}
\frac{d \bar{q}_{k}}{d s} & =\varepsilon \frac{1}{2 \pi \sqrt{a}} \sum_{n}(k \times n) \bar{q}_{k-n} \gamma_{n} \bar{q}_{n} \\
\frac{d \bar{\xi}_{k}}{d s} & =\varepsilon \frac{b^{2} \lambda_{k}^{-2}}{4 \pi \sqrt{a}} \sum_{n^{2 *}=k^{2 *}} \frac{k \times n}{n^{2_{*}}} \bar{q}_{k-n} \bar{\xi}_{n} \\
\frac{d \bar{\zeta}_{k}}{d s} & =\varepsilon \frac{b^{2} \lambda_{k}^{-2}}{4 \pi \sqrt{a}} \sum_{n^{2 *}=k^{2 *}} \frac{k \times n}{n^{2 *}} \bar{q}_{k-n} \bar{\zeta}_{n} .
\end{aligned}
$$

From the first equation we see that the evolution of the renormalized potential vorticity is given at this order simply by the quasigeostrophic equation, with no feedback from the gravity waves. The second and third equations tell us that only gravity waves belonging to the same energy shell (i.e., those having the same frequency modulus) interact, modulated by the appropriate vortical modes. This fact can be seen by noticing the skew-Hermitian nature of the operator $A_{k n}=$ const $\cdot(k \times n) \bar{q}_{k-n} / \lambda_{k}^{2} n^{2 *}$, as pointed out by Embid and Majda (1996, and references therein).

To the same order, the approximate solution for $u=$ $\left(q_{k}, \xi_{k}, \zeta_{k}\right)$ is given by the nonresonant terms in (4.9) [cf. (2.13) and recall that $u=e^{-L s} v$ ]: 


$$
\begin{aligned}
& \tilde{q}_{k}^{1}(s)=\bar{q}_{k}(s)+\varepsilon \frac{b}{2 \pi \sqrt{a}} \sum_{n}(k \times n) \bar{q}_{k-n} \cdot \frac{1}{n^{2 *}}\left(\frac{e^{-i \lambda_{n} s}-1}{\lambda_{n}} \bar{\xi}_{n}-i \frac{e^{i \lambda_{n} s}-1}{\lambda_{n}} \bar{\zeta}_{n}\right) \\
& \tilde{\xi}_{k}^{1}(s)=e^{-i \lambda_{k} s} \bar{\xi}_{k}(s)+\varepsilon \frac{b \lambda_{k}^{-2}}{4 \pi \sqrt{a}} \sum_{n}^{\prime}(k \times n) \bar{q}_{k-n}\left[\frac{1-e^{-i \lambda_{k} s}}{\lambda_{k}} \gamma_{n} \bar{q}_{n}+\frac{b}{n^{2 *}}\left(\frac{e^{i \lambda_{n} s}-e^{-i \lambda_{k} s}}{\lambda_{k}+\lambda_{n}} \bar{\zeta}_{n}-i \frac{e^{-i \lambda_{n} s}-e^{-i \lambda_{k} s}}{\lambda_{k}-\lambda_{n}} \bar{\xi}_{n}\right)\right] \\
& \tilde{\zeta}_{k}^{1}(s)=e^{i \lambda_{k} s} \bar{\zeta}_{k}(s)+\varepsilon \frac{i b \lambda_{k}^{-2}}{4 \pi \sqrt{a}} \sum_{n}^{\prime}(k \times n) \bar{q}_{k-n}\left[\frac{1-e^{i \lambda_{k} s}}{\lambda_{k}} \gamma_{n} \bar{q}_{n}-\frac{b}{n^{2 *}}\left(\frac{e^{i \lambda_{n} s}-e^{i \lambda_{k} s}}{\lambda_{n}-\lambda_{k}} \bar{\zeta}_{n}+i \frac{e^{-i \lambda_{n} s}-e^{i \lambda_{k} s}}{\lambda_{k}+\lambda_{n}} \bar{\xi}_{n}\right)\right]
\end{aligned}
$$

Here, terms with vanishing denominators are excluded in the sums over $n$ (denoted by the primes on the sum) as they are accounted for in (4.10). In this paper, we shall assume that these expressions define well-behaved functions when Fourier transformed back into physical space, that is, that terms of the form $\lambda_{k} \pm \lambda_{n}$ will not cause the Fourier series to diverge. Similar comments apply to the $O\left(\varepsilon^{2}\right)$ evolution equations (4.15) and (4.16); this is related to the small denominator problem alluded to previously.

According to (4.11a), the original potential vorticity is given by a quasigeostrophic part $\bar{q}_{k}(s)$ plus a small rapidly oscillating component. This fact has been pointed out by Embid and Majda (1996) and by Babin et al. (2000) in the contexts of the shallow-water and the three-dimensional Euler equations.

We also see that the (original) gravity waves consist of a linear oscillating part, plus a small correction that includes a slowly varying mean part determined only by the (renormalized) potential vorticity. This slow part, which to $O(\varepsilon)$ is

$$
\begin{aligned}
& \bar{\xi}_{k}^{\text {slow }}=\frac{\varepsilon b}{4 \pi \sqrt{a}} \lambda_{k}^{-3} \sum_{n}(k \times n) \bar{q}_{k-n} \gamma_{n} \bar{q}_{n} \\
& \bar{\zeta}_{k}^{\text {slow }}=\frac{\varepsilon i b}{4 \pi \sqrt{a}} \lambda_{k}^{-3} \sum_{n}(k \times n) \bar{q}_{k-n} \gamma_{n} \bar{q}_{n},
\end{aligned}
$$

corresponds precisely to the slaving relation $U(\bar{y} ; \varepsilon)$ discussed above and is therefore the leading-order balanced component of the fast variables. This tells us that at this order, the slaved and the free parts of the gravity waves are separable.

For arbitrary values of $a$, the resonant set (or, equivalently, the energy shell) may contain many (although always a finite number of) wavenumbers. In the generic case where $a$ is irrational, however, $k^{2 *}=n^{2 *}$ only when $k_{1}= \pm n_{1}$ and $k_{2}= \pm n_{2}$. Thus, each resonant set only contains these four wavenumbers, and the renormalized equations $(4.10 \mathrm{~b}, \mathrm{c})$ become

$$
\begin{aligned}
\frac{d \bar{\xi}_{k}}{d s}= & \varepsilon \frac{b^{2} \lambda_{k}^{-2}}{2 \pi a^{3 / 2}} \frac{k_{1} k_{2}}{k^{2 *}} \\
& \times\left(\bar{q}_{\left(2 k_{1}, 0\right)} \bar{\xi}_{\left(-k_{1}, k_{2}\right)}-\bar{q}_{\left(0,2 k_{2}\right)} \bar{\xi}_{\left(k_{1},-k_{2}\right)}\right) \\
\frac{d \bar{\zeta}_{k}}{d s}= & \varepsilon \frac{b^{2} \lambda_{k}^{-2}}{2 \pi a^{3 / 2}} \frac{k_{1} k_{2}}{k^{2 *}}
\end{aligned}
$$

$$
\times\left(\bar{q}_{\left(2 k_{1}, 0\right)} \bar{\zeta}_{\left(-k_{1}, k_{2}\right)}-\bar{q}_{\left(0,2 k_{2}\right)} \bar{\zeta}_{\left(k_{1},-k_{2}\right)}\right) .
$$

It can be verified from this that the energy of the quartet is conserved:

$$
\frac{d}{d s} \sum_{k} \bar{\zeta}_{k} \bar{\xi}_{-k}=0,
$$

where the sum is taken over the quartet. The quartet interactions, and their analogue in the 3D Euler equations with periodic boundary conditions (nonresonant case) where $O(\varepsilon)$ interactions are limited to octets, have been discovered by Babin et al. (2000). They also pointed out that when one-dimensional averages $q_{(k, 0)}, q_{(0, k)}$ vanish initially (in which case they are zero for all times; note that $\bar{q}_{k}=q_{k}$ at $t=0$ ), the gravity waves in this $O(\varepsilon)$ model are integrable for all times; this can be seen directly from the renormalized equations (4.10). This is a direct consequence of the geometry of the doubly periodic domain with a nonresonant aspect ratio, and is not true in general.

After some algebra, and writing $\alpha:=b / 2 \pi \sqrt{a}$, we find that the $O\left(\varepsilon^{2}\right)$ contribution of $d \bar{q}_{k} / d s$ is given by [cf. (2.18)]

$$
\begin{aligned}
W_{2}^{q_{k}}= & \sum_{m^{2 *}=n^{2 *}} \frac{\alpha^{2} b^{2}}{\lambda_{m}} \frac{k \times n}{n^{2_{*}}} \frac{(k-n) \times m}{m^{2_{*}}} \\
& \times \bar{q}_{k-n-m}\left[\bar{\zeta}_{n} \bar{\xi}_{m}-\bar{\xi}_{n} \bar{\zeta}_{m}\right] \\
& +\sum_{m, n} \frac{\alpha^{2} b}{\lambda_{m}}(k \times n)\left(\gamma_{n}-\gamma_{k-n}\right) \\
& \times \frac{n \times m}{m^{2^{*}}} \bar{q}_{k-n} \bar{q}_{n-m}\left[i \bar{\zeta}_{m}-\bar{\xi}_{m}\right] \\
& -\sum_{m, n} \frac{\alpha^{2} b}{\lambda_{n}}(k \times n) \frac{(k-n) \times m}{n^{2_{*}}} \\
& \times \gamma_{m} \bar{q}_{k-n-m} \bar{q}_{m}\left[i \bar{\zeta}_{n}-\bar{\xi}_{n}\right] \\
& -\sum_{m^{2 *}=n^{2 *}} \frac{\alpha^{2} b^{2}}{2 \lambda_{m}^{3}} \frac{k \times n}{n^{2_{*}}} \frac{n \times m}{m^{2_{*}}} \\
& \times \bar{q}_{k-n} \bar{q}_{n-m}\left[i \bar{\zeta}_{m}-\bar{\xi}_{m}\right] .
\end{aligned}
$$

By putting $\bar{\xi} \sim \bar{\zeta} \sim O(\varepsilon)$, it can be seen that this expression vanishes on the slow manifold $\mathcal{S}$. Considered together with (4.10a), this implies that there is no 


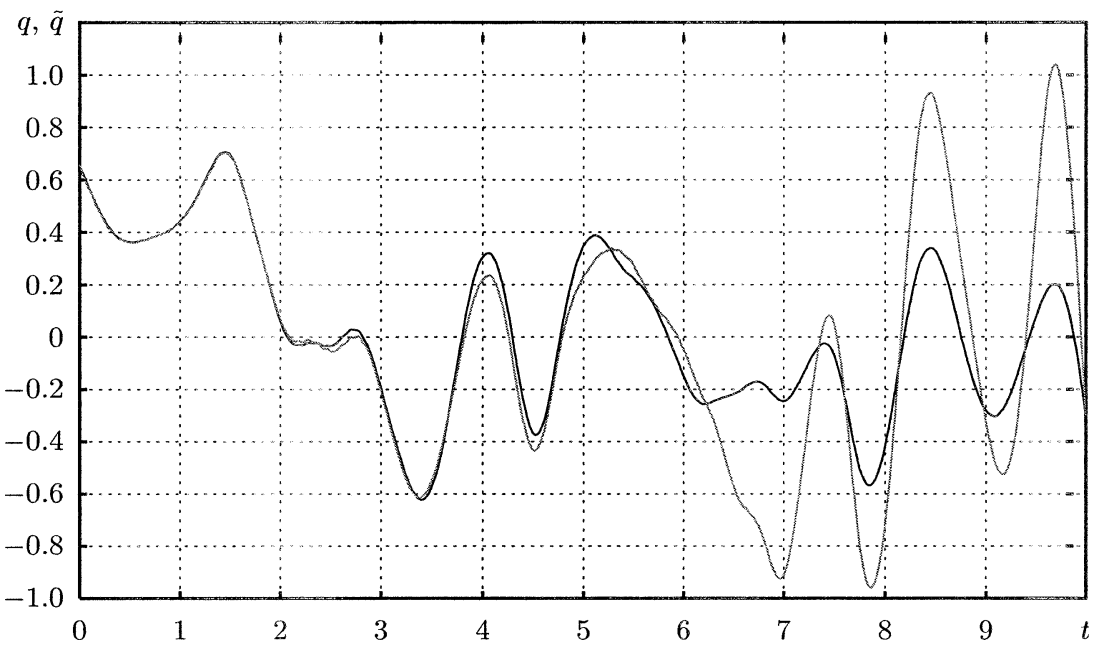

FIG. 3. The evolution of $\operatorname{Re} q_{k}$ (black line) and its renormalization approximation $\operatorname{Re} \tilde{q}_{t}$ (gray line) for $k=(2,3)$; Ro $=0.1, B=0.5$. Pointwise accuracy holds for several eddy turnaround times but eventually fails. See text in section 5 for more details.

gravity wave feedback on the balanced motion on $\mathcal{S}$ to this order.

Away from $\mathcal{S}$, however, we have not been able to find a decoupling analogous to that at $O(\varepsilon)$ above. For isolated four-wave resonances in the shallow-water equations, a careful analysis of the interaction coefficients by Callet (1997) found no mechanism that would allow finite gravity waves to influence the vortical modes in what would be equivalent to our $O\left(\varepsilon^{2}\right)$ approximation, therefore suggesting some sort of decoupling. To show that this result carries over to the full model would require an analysis of second-order equations such as (4.15).

For $d \bar{\xi}_{k} / d s$, we find

$$
\begin{aligned}
W_{2}^{\xi_{k}}= & \sum_{n^{2 *}=k^{2 *}}^{\prime} \frac{i \alpha^{2} b}{2 \lambda_{k}^{2}}\left\{\frac{k \times m}{\lambda_{k-m}} \frac{(k-m) \times n}{n^{2 *}} \gamma_{m} \bar{q}_{m} \bar{q}_{k-m-n}+\frac{k \times m}{\lambda_{n}} \frac{m \times n}{n^{2 *}} \gamma_{m} \bar{q}_{k-m} \bar{q}_{m-n}+\frac{k \times m}{\lambda_{m}} \frac{m \times n}{n^{2 *}} \frac{\bar{q}_{k-m}}{\lambda_{m}^{2}-\bar{q}_{m-n}} \lambda_{n}^{2}\right\} \bar{\xi}_{n} \\
+ & \sum_{n^{2 *} k^{2 *}}^{\prime} \frac{\alpha^{2} b}{2 \lambda_{k}^{2}} \frac{k \times n}{n^{2 *}}\left\{\frac{b}{\lambda_{m}} \frac{(k-n) \times m}{m^{2 *}} \bar{q}_{k-n-m} \bar{\xi}_{n}\left[\bar{\xi}_{m}-i \bar{\zeta}_{m}\right]\right. \\
& \left.\quad+\frac{n \times m}{2 \lambda_{n}^{2}} \bar{q}_{k-n} \bar{q}_{n-m}\left[\frac{\gamma_{m} \bar{q}_{m}}{\lambda_{n}}+\frac{b}{m^{2 *}} \frac{\bar{\zeta}_{m}}{\lambda_{n}+\lambda_{m}}+\frac{i b}{m^{2 *}} \frac{\bar{\xi}_{m}}{\lambda_{n}-\lambda_{m}}\right]\right\} \\
+ & \sum_{n, m}^{\prime} \frac{\alpha^{2}}{2 \lambda_{k}^{2}}(k \times n)\left\{\frac{m \times n}{\lambda_{k}} \gamma_{n} \gamma_{m} \bar{q}_{k-n} \bar{q}_{n-m} \bar{q}_{m}\right. \\
& \left.+((k-n) \times m) \gamma_{m} \bar{q}_{k-n-m} \bar{q}_{m}\left[-\frac{\gamma_{n} \bar{q}_{n}}{\lambda_{k}}-\frac{b}{n^{2 *}} \frac{\bar{\zeta}_{n}}{\lambda_{k}+\lambda_{n}}+\frac{i b}{n^{2 *}} \frac{\bar{\xi}_{n}}{\lambda_{k}-\lambda_{n}}\right]\right\} \\
+ & \sum_{m^{2 *}=n^{2 *}}^{\prime} \frac{\alpha^{2} b^{2}}{4 \lambda_{k}^{2} \lambda_{n}^{2}} \frac{n \times m}{m^{2 *}} \bar{q}_{k-n} \bar{q}_{n-m}\left[\frac{i \bar{\xi}_{m}}{\lambda_{k}-\lambda_{n}}-\frac{\bar{\zeta}_{m}}{\lambda_{k}+\lambda_{n}}\right] .
\end{aligned}
$$

As before, resonant sums are excluded. Again, we note that when $\bar{\xi} \sim \bar{\zeta} \sim O(\varepsilon)$, all the terms are of $O(\varepsilon)$, with the exception of the $\bar{q} \bar{q} \bar{q}$ terms (i) in the second sum, which come from the $\bar{X}_{x} \cdot X$ term in (3.21) and thus cancel when $W_{1}^{\xi_{k}}$ in (4.10b) is expanded around $\bar{\xi}=0$, and (ii) in the third sum, which come from the $X_{v} \cdot \bar{Y}$ term in (3.21) and thus cancel with $\Psi^{\prime} \cdot \bar{Y}$ in the same equation. Therefore, $\varepsilon^{2} W_{\bar{z}}^{k} \sim O\left(\varepsilon^{3}\right)$ on $\mathcal{S}$. Once again, there is no gravity wave feedback on the balanced motion on $\mathcal{S}$ to the order in question.

The corresponding expression for $W_{2}^{\xi_{k}}$ is very similar to that for $W_{2}^{\xi_{k}}$; in fact, it can be obtained using the fact 


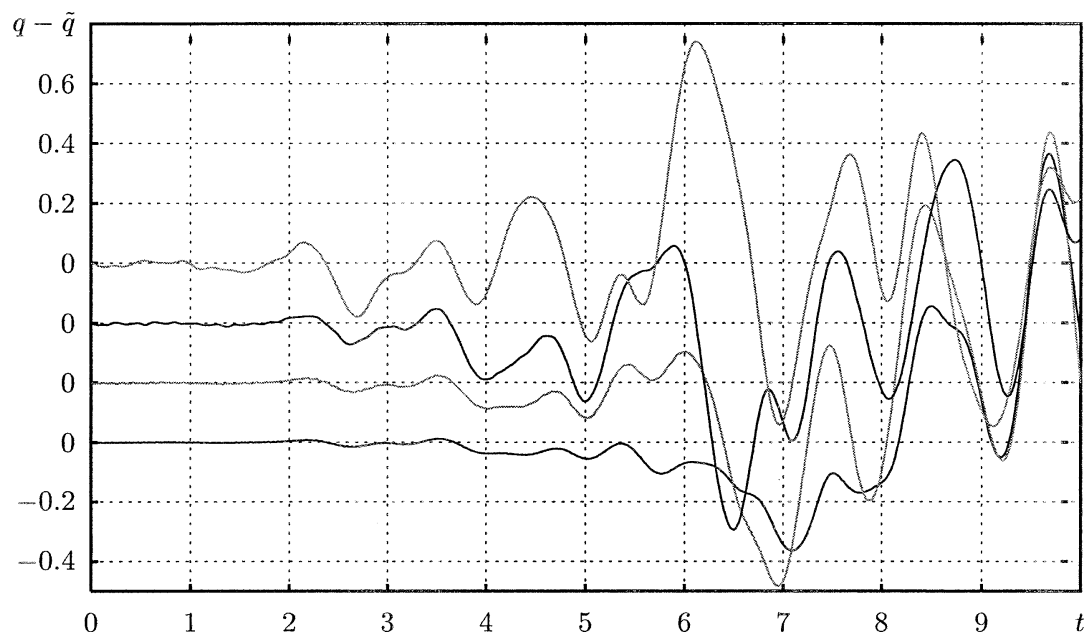

FIG. 4. $\operatorname{Re}\left(q_{k}-\tilde{q}_{k}\right)$ for (bottom to top) Ro $=0.05,0.1,0.2$, and 0.4 . In all cases $k=(2$, 3 ) and $B=0.5$. The accuracy time is seen to scale logarithmically with $\varepsilon$.

that the definitions (4.7) imply that $\bar{\zeta}_{k}=i \bar{\xi}_{-k}^{*}$. There is thus no need to discuss it separately.

\section{Numerical examples}

A (pseudo)spectral truncation of the weak-wave model (4.5) is integrated in time, along with its renormalized counterpart (4.10). As our purpose here is to illustrate the method, a low truncation with $17^{2}$ wavenumbers is used. A nonresonant domain aspect ratio $a \simeq 0.618034$ is used. In all the simulations, we take as initial conditions

$$
\left|q_{k}\right| \propto k^{m} e^{-m k k k_{0}},
$$

where $m=8$ and $k_{0}=4$ and the phases of the (complex) $q_{k}$ are chosen at random. Similar initial conditions are used for the ageostrophic variables. The initial geostrophic energy

$$
E_{g}=\sum_{k} \gamma_{k}\left|q_{k}\right|^{2}
$$

is set to be 1 (this fixes the normalization constant for $q$ above), and the initial energy in the ageostrophic modes is fixed to be $1 / 3$.

To $O(\varepsilon)$, the renormalization procedure aims to approximate the solution of the original equations of motion (4.5) by the solution of the renormalized evolution equation (4.10) using the relations (4.11). There are (at least) two issues of interest here. The first is pointwise accuracy-important in short-range weather forecasting - where one directly compares the solution $v(s)$ with its approximation $\tilde{v}^{1}(s)=R(\bar{v}(s), s)$. The second, more difficult, issue concerns comparison of qualitative behavior over longer timescales, which is of importance in, say, climate modeling.

As the system we are dealing with is nonintegrable and even chaotic, it is clear that one cannot expect pointwise accuracy for arbitrary initial conditions beyond the timescale of the largest Lyapunov exponent present in the system (either the original or the renormalized one), which is typically of the order of the eddy turnaround time, namely $t \sim O(1)$. As (2.21) tells us, one can increase the accuracy significantly for a fixed time by going to higher order in $\varepsilon$, but since the error grows exponentially, the validity time only increases logarithmically with $1 / \varepsilon$.

Keeping this caveat in mind we turn to Fig. 3, where we plot (the real part of) the solution of the full problem $q_{k}(t)$ and its approximation $\tilde{q}_{k}^{1}(t)$ for $k=(2,3)$. Here we have taken $B=0.5$ and Ro $=0.1$, giving $\varepsilon \simeq 0.045$. It can be seen that for several eddy turnaround times, the approximation is a good one, but it inevitably diverges from the true solution over longer times.

In Fig. 4 we plot the difference between the two curves in Fig. 3 for different values of Ro (and thus of $\varepsilon)$. Here the logarithmic dependence of the accuracy time with $\varepsilon$ as given in (2.21) becomes apparent: halving $\varepsilon$ only appears to increase the accuracy time by a fixed amount, approximately $\Delta t \sim 1$.

One qualitative property of the renormalized system is given by (4.14), which says that the ageostrophic energy

$$
E_{\mathrm{ag}, k}=\frac{1}{k^{2 *}}\left(\left|\Delta_{k}\right|^{2}+\lambda_{k}^{2}\left|h_{k}\right|^{2}\right)
$$

is conserved within each energy shell. In the top panel of Fig. 5, we plot $E_{\mathrm{ag}, k}$ of the mode $k=(2,3)$ for different values of Ro, and in the bottom panel we plot $\Sigma_{k} E_{\text {ag, } k}$, where the sum is taken within the energy shell. Note the much longer integration time than in Fig. 4. At least while our approximation is formally valid, $\Sigma_{k} E_{\mathrm{ag}, k}$ differs from $\Sigma_{k} \bar{\zeta}_{k} \bar{\xi}_{-k}$ by a (rapidly oscillating) quantity of order $\varepsilon$ - given by the $O(\varepsilon)$ terms of $(4.11 \mathrm{~b}, \mathrm{c})$, which we do not compute here. Part of this difference appears as the rapid oscillations seen in the bottom curve in the 

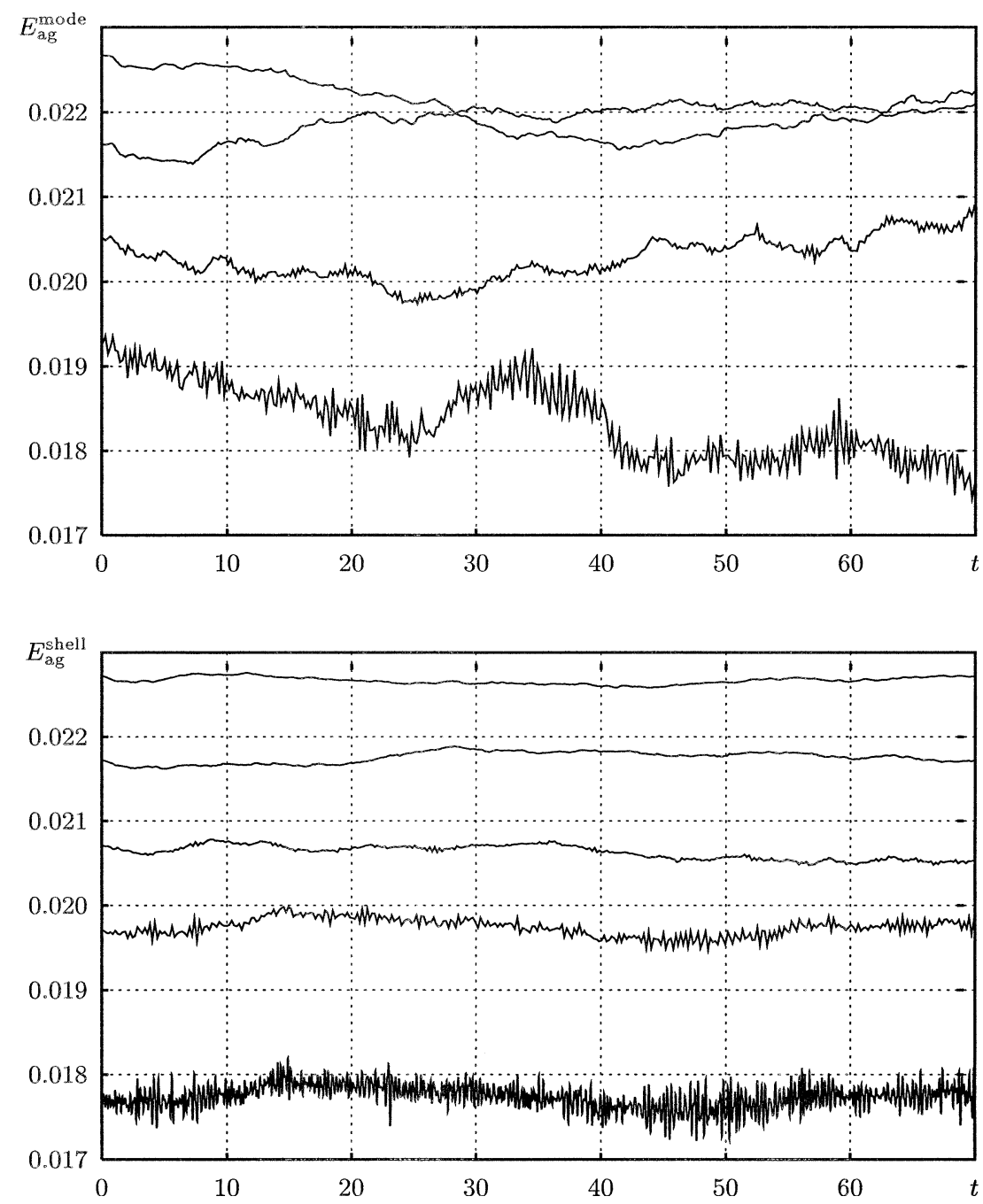

FIG. 5. Plot of the ageostrophic energy $E_{\mathrm{ag}}=E-E_{\mathrm{g}}$ as a function of time $t$ : (top) values of $4 E_{\mathrm{ag}}$ for the mode $k=(2,3)$; the curves correspond to (top to bottom) Ro $=0.05,0.1$, 0.2 , and 0.4 , each shifted vertically by 0.001 to improve visual clarity (the factor of 4 is to facilitate comparison with the bottom panel). (bottom) Values of $E_{\mathrm{ag}}$ for the shell with $k^{2_{*}}=|(2,3)|^{2_{*}}$; the curves correspond to (top to bottom) Ro $=0.05,0.1,0.2$, and 0.4, smoothed in $t$ and shifted vertically as above. All curves have been smoothed by averaging over the period of the fast oscillations, except for the bottom curve, which is the unsmoothed curve for Ro $=0.4$.

bottom panel of Fig. 5. Applying a time smoothing takes care of these rapid oscillations, giving the smoother (upper) curves seen in the bottom panel of Fig. 5. [Note that we still have the slowly varying part of $(4.11 b, c)$, but we know that this is of $O(\varepsilon)$.]

It can be seen that $E_{\mathrm{ag}}^{\text {shell }}$ does indeed evolve very slowly, varying by less than $1 \%$ or $2 \%$ over many tens of eddy turnaround times - far beyond the timescale of validity of the approximation. In this sense we can say that the approximate system predicts some qualitative behavior of the parent (full) system.

It is possible that other qualitative properties may hold over long times. A good way to find them is by careful scrutiny of the renormalized evolution equations such as (4.15), (4.16), and their higher-order analogues.

\section{Discussion}

Compared with traditional balance models, the advantage of the present approach is clear: it allows us to include the effect of free gravity waves ("unbalanced motion") in the dynamics while still preserving the slow nature of the approximating model. As with any approximation method applied to nontrivial dynamics, we find pointwise accuracy to fail after a short time (which is largely independent of the approximation method), as 
illustrated in Figs. 3 and 4. This is also true for classical balanced dynamics: unless the balance condition is satisfied exactly by the full dynamics, a trajectory of the balance model will generically diverge from the true trajectory even if it stays near the hypothetical "balance manifold," because of chaotic slow dynamics.

We have also seen that the balanced solution obtained using the slaving approach (Warn et al. 1995) can be seen as a natural special (slow) solution within our general framework. This allows us to gain insight into the dynamics in the neighborhood of the slaving manifold (as well as far away from it), giving us, among other things, the stability of the slow solution over timescales $t \sim O(1)$. [Such validity estimates can also be obtained independently - cf. the appendix of Wirosoetisno and Shepherd (2000) for general finite-dimensional systems, and Jones (2002) for the shallow-water equations.] Renormalization also provides a natural way to remove the blow up of the solution of a naïve slaving expansion, justifying the use of unexpanded slow variables in Warn et al. (1995).

For long-term qualitative studies, balance models are regarded to be useful because the full system tends to stay almost balanced for long times, and thus the solution of the balance model will presumably share many qualitative properties of the full solution. Although this assumption is borne out by (indeed, was originally born of) observations as well as numerical experiments, no rigorous justification has been offered as to why this should be the case.

In the context of the weak-wave model, our numerical results suggest a more general behavior: the ageostrophic energy remains largely confined within energy shells for long times. As with the balance assumption above, this appears to imply that the energy shells form a family of very stable manifolds ("fuzzy layers" is perhaps a more appropriate term), which is traversed by the full dynamics only over very long timescales. In this picture, the slow manifold is the innermost of this family of manifolds (cf. Bokhove and Shepherd 1996). Thus, in yet another way, classical balanced dynamics can be seen as a special case of the renormalized dynamics.

From the point of view of dynamical systems, this picture reminds one of the family of adiabatic invariant surfaces present in many canonical Hamiltonian systems with a separation of timescales. Unlike in general dynamical systems, the timescale of validity in this case can often be exponentially long in $\varepsilon$ (Wirosoetisno and Shepherd 2000, and references therein). It would be interesting to investigate if and how the noncanonical Hamiltonian (Poisson) structure of the weak-wave model has anything to do with the behavior we see above.

Finally, we would like to emphasize that the ideas of averaging and renormalization as presented in section 2 of this paper are certainly not new, as they date back at least to the 1930s and 1940s. There have also been papers in which the method is applied to problems in GFD, albeit only to first order (e.g., Embid and Majda
1996; Chemin 1997; Gallagher 1998; Babin et al. 2002). What we aim to do here is bring the method closer to the atmospheric audience by avoiding the functional analytic aspects and describing the procedure as clearly as possible. The connection with slaving (section 3 ), on the other hand, appears not to have been pointed out before; in doing so we hope to put the general renormalized/averaged approximate solution in context as a natural extension of the classical balance ideas.

Acknowledgments. The work reported in this paper has received support from the Natural Sciences and Engineering Research Council and the Meteorological Service of Canada (TGS), the Engineering and Physical Sciences Research Council (DW), the National Science Foundation (Grant DMS-0074334, RMT), and the Research Fund of Indiana University (RMT and DW). We thank the reviewers for their thorough scrutiny and constructive criticisms, which have led to improvements in the manuscript.

\section{REFERENCES}

Allen, J. S., 1993: Iterated geostrophic intermediate models. J. Phys. Oceanogr., 23, 2447-2461.

Babin, A., A. Mahalov, and B. Nicolaenko, 2000: Fast singular oscillating limits and global regularity for the $3 \mathrm{~d}$ primitive equations of geophysics. Modél. Math. Anal. Num., 34, 201-222.

,$- \ldots$, and ——, 2001: 3d Navier-Stokes and Euler equations with initial data characterized by uniformly large vorticity. Indiana Univ. Math. J., 50, 1-35.

,-- , and,- 2002 : Fast singular oscillating limits of stablystratified three-dimensional Euler-Boussinesq equations and ageostrophic wave fronts. Large-Scale Atmosphere-Ocean Dynamics I, J. Norbury and I. Roulstone, Eds., Cambridge University Press, 126-201.

Baer, F., and J. J. Tribbia, 1977: On complete filtering of gravity modes through nonlinear initialization. Mon. Wea. Rev., 105, 1536-1539.

Bogolyubov, N. N., and Yu. A. Mitropol'skii, 1958: Asymptotic Methods in the Theory of Non-linear Oscillations. Hindustan, $537 \mathrm{pp}$.

Bokhove, O., and T. G. Shepherd, 1996: On Hamiltonian balanced dynamics and the slowest invariant manifold. J. Atmos. Sci., 53, $276-297$.

Bruno, A. D., 1989: Local Methods in Nonlinear Differential Equations. Springer-Verlag, $348 \mathrm{pp}$.

Callet, J. M., 1997: Long time asymptotics for fast wave averaging of the rotating shallow water equations. Ph.D. thesis, Princeton University, $152 \mathrm{pp}$.

Charney, J. G., 1948: On the scale of atmospheric motions. Geofys. Publ., 17, 3-17.

_ 1955: The use of the primitive equations of motion in numerical prediction. Tellus, 7, 22-26.

Chemin, J.-Y., 1997: À propos d'un problème de pénalisation de type antisymmétrique. J. Math. Pures Appl., 76, 739-755.

Chen, L.-Y., N. Goldenfeld, and Y. Oono, 1996: Renormalization group and singular perturbations: Multiple scales, boundary layers, and reductive perturbation theory. Phys. Rev., E54, 376394.

Embid, P. F., and A. J. Majda, 1996: Averaging over fast gravity waves for geophysical flows with arbitrary potential vorticity. Comm. P.D.E., 21, 619-658.

_ , and —_, 1998: Low Froude number limiting dynamics for stably stratified flow with small or finite Rossby number. Geophys. Astrophys. Fluid Dyn., 87, 1-50. 
Farge, M., and R. Sadourny, 1989: Wave-vortex dynamics in rotating shallow water. J. Fluid Mech., 206, 433-462.

Ford, R., 1994a: Gravity wave radiation from vortex trains in rotating shallow water. J. Fluid Mech., 281, 81-118.

_ 1994b: The instability of an axisymmetric vortex with monotonic potential vorticity in rotating shallow water. J. Fluid Mech., 280, 303-334.

$\ldots, 1994 \mathrm{c}$ : The response of a rotating ellipse of uniform potential vorticity to gravity wave radiation. Phys. Fluids, 6, 3694-3704.

Gallagher, I., 1998: Asymptotic of the solutions of hyperbolic equations with a skew-symmetric perturbation. J. Diff. Eq., 150,363384.

Goldenfeld, N., 1992: Lectures on Phase Transitions and the Renormalization Group. Addison-Wesley, 394 pp.

Jones, D. A., 2002: Mathematical analysis of geophysical balance models. J. Diff. Eq., 179, 1-26.

Kreiss, H.-O., 1979: Problems with different time scales for ordinary differential equations. SIAM J. Numer. Anal., 16, 980-998.

Lorenz, E. N., 1980: Attractor sets and quasi-geostrophic equilibrium. J. Atmos. Sci., 37, 1685-1699.

_ 1992: The slow manifold-What is it? J. Atmos. Sci., 49, 24492451.

—_, and V. Krishnamurthy, 1987: On the nonexistence of a slow manifold. J. Atmos. Sci., 44, 2940-2950.

Machenhauer, B., 1977: On the dynamics of gravity oscillations in a shallow water model, with applications to normal mode initialization. Beitr. Phys. Atmos., 50, 253-271.

Majda, A. J., and P. F. Embid, 1998: Averaging over fast gravity waves for geophysical flows with unbalanced initial data. Theor. Comput. Fluid Dyn., 11, 155-169.

McIntyre, M. E., and W. A. Norton, 2000: Potential vorticity inversion on a hemisphere. J. Atmos. Sci., 57, 1214-1235.

Moise, I., and R. M. Temam, 2000: Renormalization group method. Application to Navier-Stokes equations. Discrete Contin. Dyn. Syst., 6, 191-210.

— , and M. Ziane, 2001: Renormalization group method. Applications to partial differential equations. J. Dyn. Diff. Eq., 13, 275-321.
E. Simonnet, R. Temam, and M. Ziane, 1998: Numerical simulation of differential systems displaying rapidly oscillating solutions. J. Eng. Math., 34, 201-214.

Nayfeh, A. H., 1973: Perturbation Methods. Wiley, 425 pp.

Nore, C., and T. G. Shepherd, 1997: A Hamiltonian weak-wave model for shallow-water flow. Proc. Roy. Soc. London, A453, 563580.

Pedlosky, J., 1987: Geophysical Fluid Dynamics. 2d ed. SpringerVerlag, $710 \mathrm{pp}$.

Polvani, L. M., J. C. McWilliams, M. A. Spall, and R. Ford, 1994: The coherent structures of shallow-water turbulence: Deformation-radius effects, cyclone/anticyclone asymmetry and gravitywave generation. Chaos, 4, 177-186.

Schochet, S., 1994: Fast singular limits of hyperbolic PDEs. J. Diff. Eq., 114, 476-512.

Temam, R., and D. Wirosoetisno, 2002: Averaging of differential equations generating oscillations and an application to control. J. Appl. Math. Optim., in press.

Vautard, R., and B. Legras, 1986: Invariant manifolds, quasi-geostrophy and initialization. J. Atmos. Sci., 43, 565-584.

Warn, T., 1997: Nonlinear balance and quasi-geostrophic sets. Atmos.-Ocean, 35, 135-145.

—, O. Bokhove, T. G. Shepherd, and G. K. Vallis, 1995: Rossby number expansions, slaving principles, and balance dynamics. Quart. J. Roy. Meteor. Soc., 121, 723-739.

Wirosoetisno, D., 1999: Balance dynamics and stability of vortical flows. Ph.D. thesis, University of Toronto, $150 \mathrm{pp}$.

— , and T. G. Shepherd, 2000: Averaging, slaving and balance dynamics in simple atmospheric models. Physica D, 141, 3753 .

Yavneh, I., and J. C. McWilliams, 1994: Breakdown of the slow manifold in the shallow-water equations. Geophys. Astrophys. Fluid Dyn., 75, 131-161.

Yuan, L., and K. Hamilton, 1994: Equilibrium dynamics in a forceddissipative $f$-plane shallow-water system. J. Fluid Mech., 280, 369-394.

Ziane, M., 2000: On a certain renormalization group method. J. Math. Phys., 41, 3290-3299. 